\title{
Preclinical assessment of CNS drug action using eye movements in mice
}

\author{
Hugh Cahill, ${ }^{1}$ Amir Rattner, ${ }^{1}$ and Jeremy Nathans ${ }^{1,2,3,4}$ \\ ${ }^{1}$ Department of Molecular Biology and Genetics, ${ }^{2}$ Howard Hughes Medical Institute, ${ }^{3}$ Department of Ophthalmology, and \\ ${ }^{4}$ Department of Neuroscience, Johns Hopkins University School of Medicine, Baltimore, Maryland, USA.
}

\begin{abstract}
The drug development process for CNS indications is hampered by a paucity of preclinical tests that accurately predict drug efficacy in humans. Here, we show that a wide variety of CNS-active drugs induce characteristic alterations in visual stimulus-induced and/or spontaneous eye movements in mice. Active compounds included sedatives and antipsychotic, antidepressant, and antiseizure drugs as well as drugs of abuse, such as cocaine, morphine, and phencyclidine. The use of quantitative eye-movement analysis was demonstrated by comparing it with the commonly used rotarod test of motor coordination and by using eye movements to monitor pharmacokinetics, blood-brain barrier penetration, drug-receptor interactions, heavy metal toxicity, pharmacologic treatment in a model of schizophrenia, and degenerative CNS disease. We conclude that eyemovement analysis could complement existing animal tests to improve preclinical drug development.
\end{abstract}

\section{Introduction}

The development of drugs for CNS indications by the pharmaceutical industry generally follows a path from biochemical or cellbased assays to testing in animal models to human clinical trials. Attrition is high at each step. In particular, only $8 \%$ of CNS drug candidates that enter clinical trials win FDA approval, among the lowest success rates of any therapeutic area (1). The majority of these drug candidates fail in late-stage clinical trials, with attrition most commonly caused by inefficacy or undesirable side effects. This low success rate is due in part to the limited predictive power of animal testing for human CNS pharmacology (2). Additionally, drug candidates with peripheral targets can be lost from the development pipeline if they produce unwanted CNS side effects (e.g., ref. 3). Thus, there is a clear need to develop improved physiological and behavioral tests in animals that accurately predict human responses to CNS-active drugs $(4,5)$.

In rodents, functional tests that assess CNS drug action can be roughly divided into 4 categories based on behavioral complexity: (a) simple stimulus-response paradigms, such as the startle reflex, prepulse inhibition, paw withdrawal from a hot plate, or vocalization after isolation; (b) stereotyped motor tasks, such as balancing on a rotarod; (c) complex innate behaviors, such as circadian entrainment or open-field activity; and (d) learned responses, such as maze running $(6,7)$. Tests in the first 2 categories can be performed rapidly with simple equipment and can measure a variety of changes in CNS function, such as sedation, analgesia, and ataxia. By contrast, tests in the third and fourth categories require more extensive monitoring or preliminary training trials, constraints that substantially increase the time and expense of animal testing (8-10). For tests with a large volitional component, trial-totrial variability is generally large, and data sets must be averaged over many trials.

Eye movements represent a readily monitored behavior that reflects a complex set of sensory-motor computations (11). In nonfoveate mammals, such as mice, there are 3 primary types of eye movements: (a) the optokinetic reflex (OKR), (b) the vestibu-

Conflict of interest: The authors have declared that no conflict of interest exists. Citation for this article: J Clin Invest. 2011;121(9):3528-3541. doi:10.1172/JCI45557. lar-ocular reflex (VOR), and (c) spontaneous eye movements. The OKR is an involuntary response to a moving visual stimulus that consists of a series of slow eye-tracking movements (ETMs) interrupted at regular intervals by rapid resetting movements (saccades) in the opposite direction. Foveate mammals, such as humans and other primates, have additional types of eye movements, most prominently, fixational eye movements that bring the object of regard onto the fovea and maintain it there.

Eye movements are relatively unexplored in the context of preclinical CNS drug testing, although it has long been appreciated that some CNS-active drugs - including benzodiazepines, opiates, antipsychotics, and anticonvulsants - affect ETMs in humans (11-14). In the context of clinical pharmacology, one of the bestknown uses of human eye movement analysis is for monitoring toxicity in patients taking phenytoin, an antiseizure medication with a relatively narrow therapeutic window $(15,16)$. In rodents, eye movements can be recorded from an animal that is head restrained using an infrared camera to monitor the position of the pupil relative to a stationary corneal reflection (17).

As a behavioral measure of CNS activity, eye movements have several favorable attributes: they are rapid, they do not require training, and they show little or no adaptation. An OKR data set, with hundreds of saccades, can be recorded in a single 30-minute session (17-19). Like the electrocardiogram or the electroencephalogram, an eye-movement record lends itself to relatively simple and semiautomated analyses of peak size, shape, polarity, and spacing.

In this paper, we explore the use of eye-movement analysis for monitoring CNS drug action in mice. We find that many CNS-active drugs elicit distinctive and characteristic eye-movement responses that can be analyzed quantitatively. We demonstrate the use of eye-movement analysis in this context by comparing it with the commonly used rotarod test of motor coordination and by using eye movements to monitor pharmacokinetics, blood-brain barrier (BBB) penetration, drug-receptor interactions, pharmacologic treatment in a model of schizophrenia, and degenerative CNS disease.

\section{Results}

OKR responses to diverse psychoactive drugs. To rapidly assess the extent to which a variety of CNS-active drugs might affect eye movements 

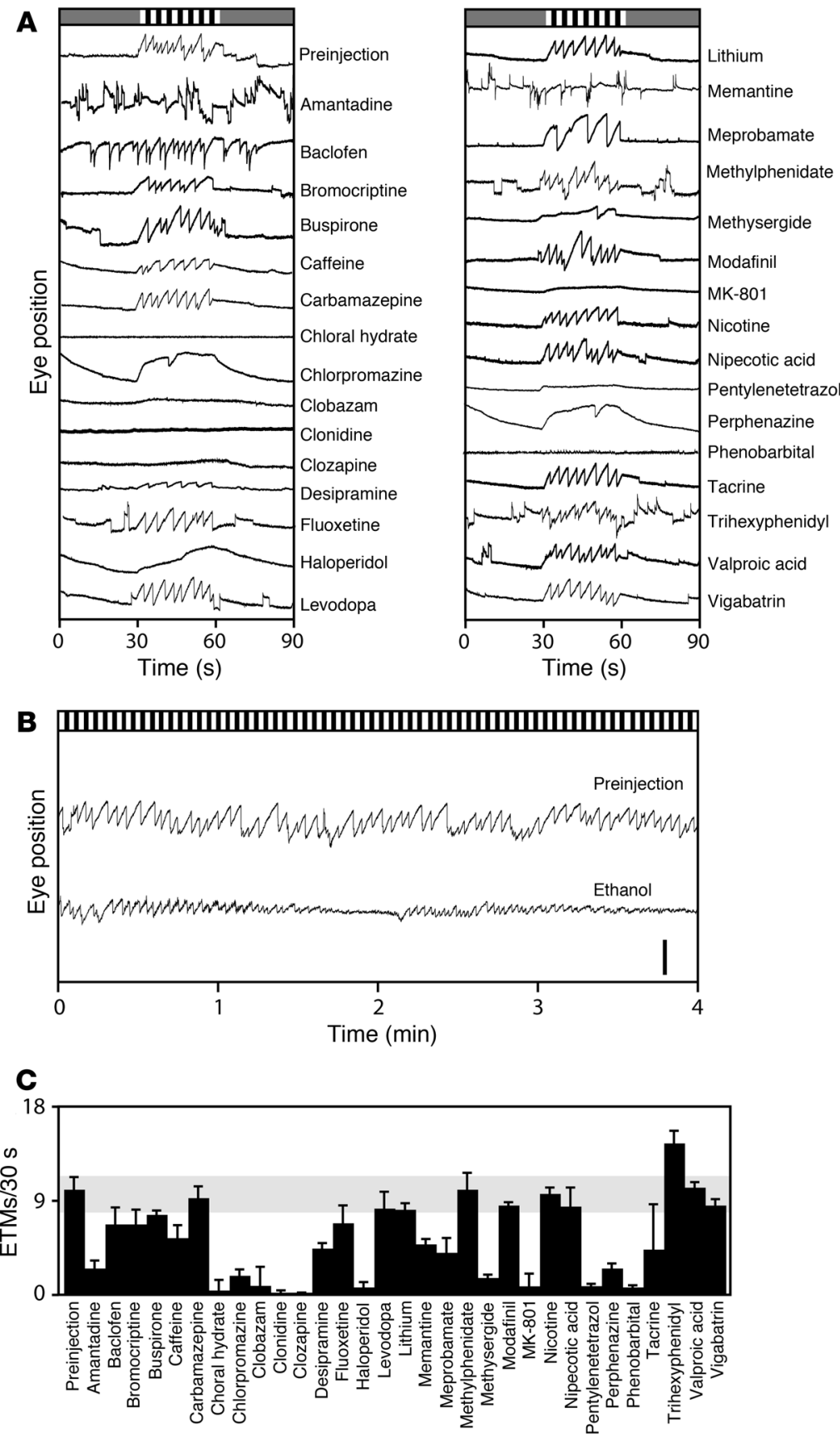
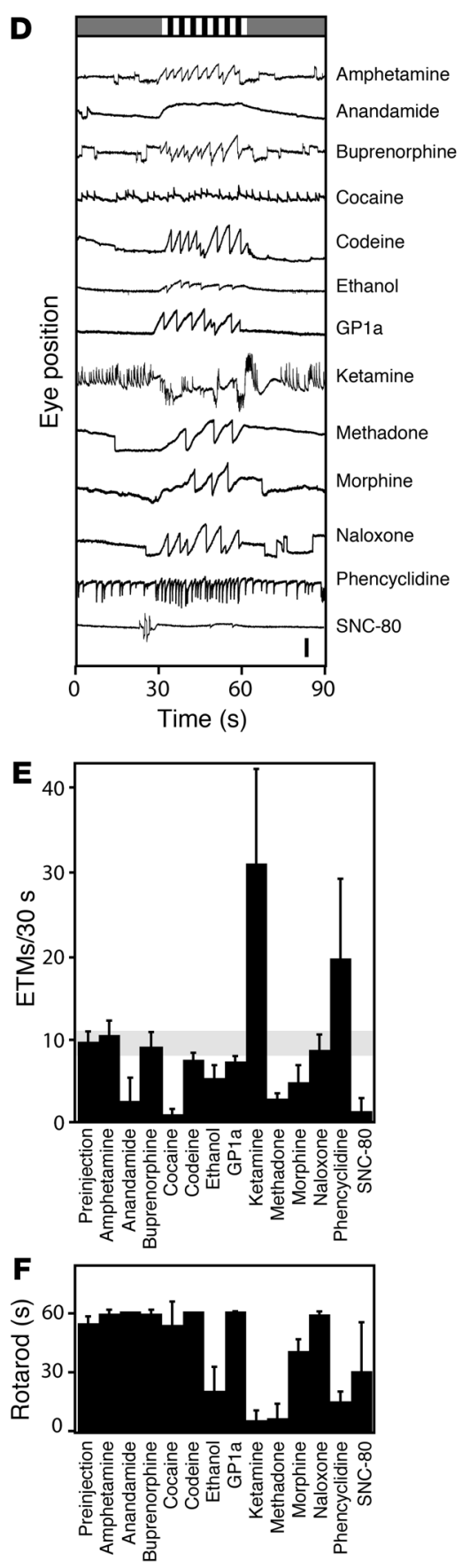

Figure 1

Survey of drug effects on the OKR. Drugs were delivered by i.p. injection at the doses listed in Supplemental Tables 1 and 2. (A-C) Medicinal and miscellaneous psychoactive compounds. (A) Representative 90-second OKR responses to 30 seconds of rotating black and white stripes, preceded and followed by 30 seconds of a uniform gray. Visual stimuli are represented schematically at the top of each panel. Scale bar: $0.5 \mathrm{~mm}$. (B) Four-minute OKR record with continuously rotating black and white stripes after i.p. injection of $2 \mathrm{~g} / \mathrm{kg}$ ethanol as a $25 \%$ ethanol solution in PBS, i.e., equivalent to a blood alcohol level of approximately $0.2 \%$. Scale bar: $1 \mathrm{~mm}$. (C) Quantification of ETM $\mathbf{B}_{30}$ during the moving stimulus interval. (D-F) Drugs of abuse and related compounds. (D and E) Representative 90-second OKR records and their quantification, as described for $\mathbf{A}$ and $\mathbf{C}$. Scale bar: $0.5 \mathrm{~mm}$. (F) Rotarod performance. For this and all other rotarod experiments, mice were given the same drug doses as shown for the OKR. In most cases, mice were tested immediately after OKR testing. Rotarod performance is quantified as the time to fall off a cylinder rotating at 7 revolutions per minute. Trials were terminated at 60 seconds. Between 2 and 7 mice were tested per drug. Data are presented as the mean \pm standard deviation. 

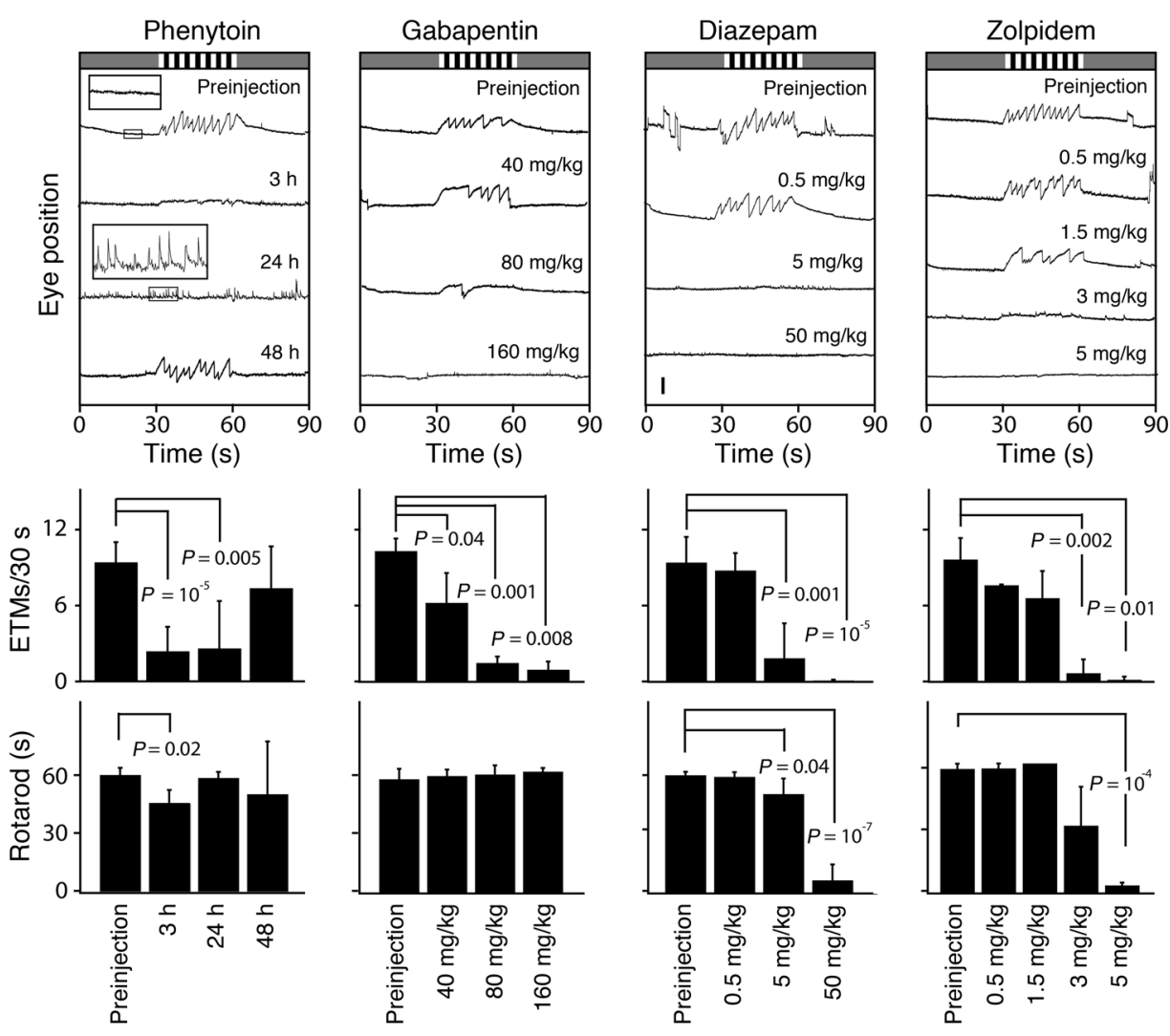

Figure 2

Quantifying the dose response and time course of drug action. OKR and rotarod performance at the indicated times after a single i.p. injection of 85 $\mathrm{mg} / \mathrm{kg}$ phenytoin or approximately 30-60 minutes after the indicated doses of gabapentin, diazepam, or zolpidem. Top panels show representative 90-second OKR records; center panels quantify the $\mathrm{ETM}_{30}$ during the rotating visual stimulus interval; and bottom panels quantify rotarod performance, as described in Figure 1. For phenytoin, boxed regions from the preinjection and 24-hour postinjection OKR records are enlarged 4 fold and shown as insets. The number of mice tested per time point or drug concentration is as follows: phenytoin, 3-6 mice; gabapentin, 3 mice; diazepam, 3-5 mice; and zolpidem, 3 mice. In the histograms, each $\mathrm{ETM}_{30}$ and rotarod data point was compared by $t$ test to the preinjection control; only those comparisons with $P<0.05$ are shown. $P$ values of less than $10^{-4}$ are rounded to the nearest factor of 10 . Scale bar: $0.5 \mathrm{~mm}$. Data are presented as the mean \pm standard deviation. in mice, we examined spontaneous and visual stimulus-induced eye movements after i.p. injection of 48 drugs (Figures 1 and 2 and Supplemental Tables 1 and 2; supplemental material available online with this article; doi:10.1172/JCI45557DS1). In this initial survey, relatively high doses were chosen to increase the likelihood that a drug effect might be observed. For this and all subsequent analyses we have used a custom-built eye-tracking apparatus in which a rotating pattern of vertical black and white stripes is projected onto the inner wall of a test cylinder from a ceiling-mounted rotating liquid crystal display projector (19). Pupil position was sampled at $60 \mathrm{~Hz}$ with an infrared video camera.

In Figure 1 and in many of the following figures, we show a representative 90 -second OKR record that encompasses 3 contiguous 30 -second segments. During the first and third segments, the wall of the cylindrical test chamber was illuminated with a uniform gray light (referred to as the null stimulus or rest period), and, during the second segment, the wall was illuminated with slowly rotating vertical black and white stripes, producing a strong stimulus for the OKR. For quantification of the number of ETMs per 30 seconds (referred to hereafter as $\mathrm{ETM}_{30}$ ), responses were typically averaged from 12 or more 30 -second visual stimulus intervals per mouse.

At the doses used in Figures 1 and 2, 31 out of 48 drugs perturbed eye movements. Injection of PBS had no effect (Supplemental Figure 1). Among medicinal drugs (Figure 1, A and C, and Figure 2), antipsychotics (chlorpromazine, clozapine, haloperidol, and perphenazine) and sedatives (chloral hydrate, phenobarbital, and the benzodiazepines, diazepam, clobazam, and zolpidem) consistently slowed or eliminated the OKR, as did the NMDA antagonist MK-801 and the antiseizure drugs gabapentin and phenytoin. The tricyclic antidepressant desipramine and the antimigraine drug methysergide reduced OKR frequency and amplitude. At $50 \mathrm{mg} / \mathrm{kg}$, pentylenetetrazol (PTZ), a GABA(A) receptor antagonist and CNS stimulant, eliminates eye movements; at higher doses, PTZ induces seizures.

Interestingly, a number of drugs induced spontaneous eye movements, defined as movements occurring during the 30-second rest periods in Figure 1A. This group includes amantadine and trihexyphenidyl, anticholinergic compounds used in the treatment of Parkinson disease; baclofen, a GABA-B receptor agonist used in the treatment of spasticity; and memantine, a low-affinity NMDA, $5 \mathrm{HT} 3$, and nicotinic acetylcholine receptor antagonist used in the treatment of Alzheimer disease. Relative to the spontaneous eye movements observed in control OKR traces, spontaneous eye movements induced by drug treatment exhibited a high frequency and a more rapid return to baseline.

For drugs of abuse and related compounds, we tested both the OKR and rotarod performance (Figure 1, B and D-F; for quantification of rotarod performance, rotarod balancing times up to 60 seconds were typically averaged from 8 or more tests per mouse). The opiates methadone and morphine slowed the OKR and produced a similar decrement in rotarod performance. SNC-80, a selective delta opioid agonist, eliminated the OKR and substantially impaired rotarod performance. Codeine, a relatively lowpotency morphine prodrug; naloxone, an opioid antagonist; and buprenorphine, a mixed opioid agonist/antagonist, had little or no effect on the OKR or on rotarod performance. Anandamide, the endogenous agonist for cannabinoid receptors CB1 and CB2, greatly slowed the OKR but had no effect on rotarod performance. By contrast, the synthetic CB2-selective agonist, GP 1a, has minimal effects on either eye movements or rotarod performance. 

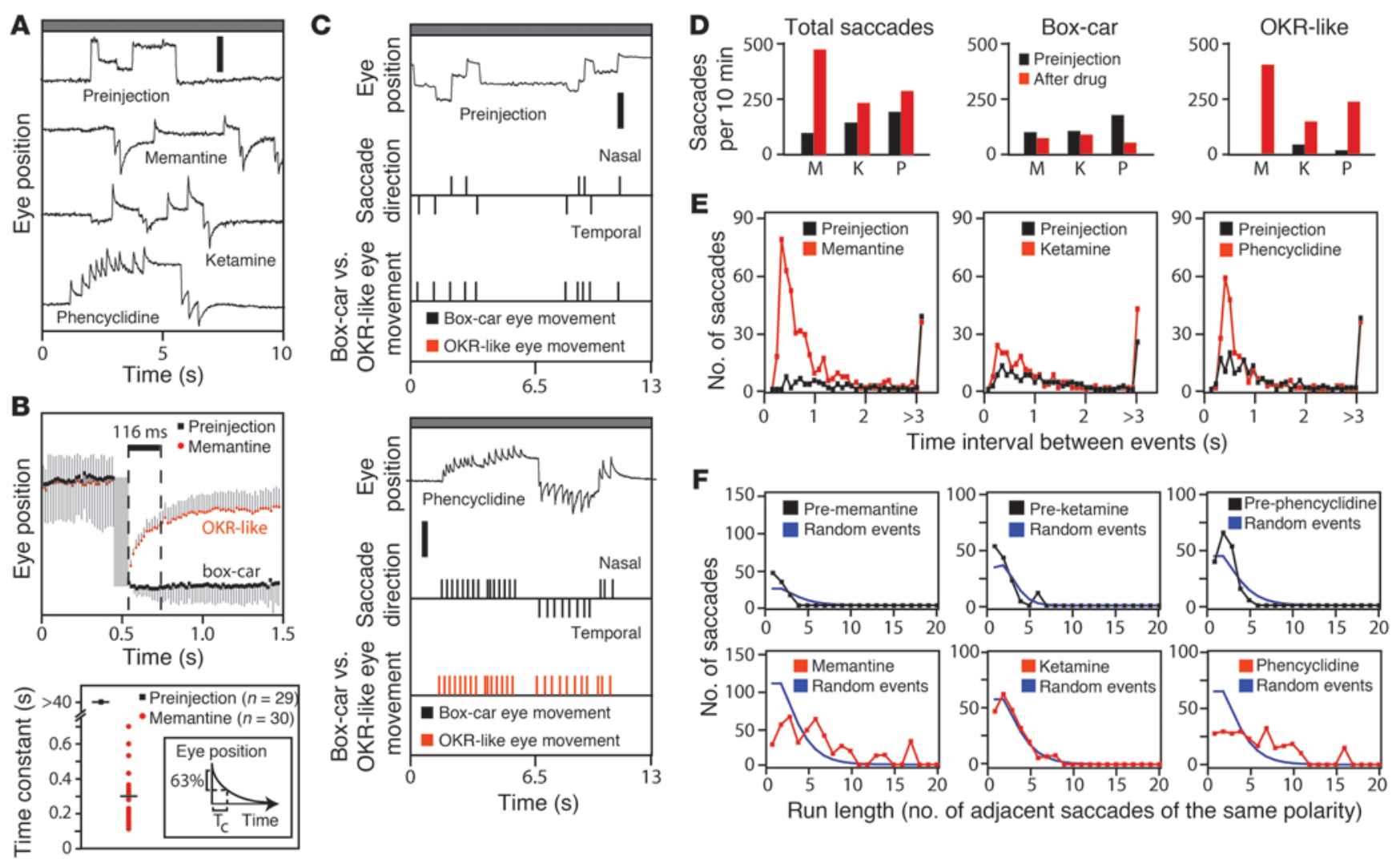

Figure 3

Drug-induced OKR-like responses in the absence of a moving visual stimulus. (A) Eye movements in the absence of a moving visual stimulus before and after an i.p. injection of ketamine ( $8 \mathrm{mg} / \mathrm{kg})$, memantine $(23 \mathrm{mg} / \mathrm{kg})$, or phencyclidine $(4 \mathrm{mg} / \mathrm{kg})$. Scale bar: $0.5 \mathrm{~mm}$. (B) Spontaneous eye movements before $(n=29)$ and after $(n=30)$ i.p. memantine, with saccade amplitudes normalized and traces temporally aligned by the saccade, and the time constant $\left(T_{C}\right)$ of the best-fitting exponential for each recording of eye position versus time for the 116 milliseconds immediately after the saccade. The inset shows a graphical definition of $\mathrm{T}_{\mathrm{C}}$ as the time corresponding to a $63 \%$ change in eye position, following exponential kinetics. Horizontal bars represent the mean. (C) Automated identification of box-car (black) versus OKR-like (red) spontaneous eye movements in 2 thirteen-second recordings, with or without i.p. phencyclidine. For each recording, the timing and polarity of the fast components were scored (central quantized trace), and then each saccade was categorized as box-car or OKR-like (bottom quantized trace). Scale bar: $1 \mathrm{~mm}$. (D) Total (left), box-car-shaped (center), and OKR-like (right) saccades during a 10-minute recording obtained before (black) or after (red) the indicated drug. $\mathrm{M}$, memantine; K, ketamine; $\mathrm{P}$, phencyclidine. (E) Distribution of time intervals between adjacent fast components before versus after drug administration. (F) Clustering of saccade polarity: the number of adjacent saccades of the same polarity within a 10-minute interval before versus after drug administration. Blue lines show the calculated distribution of adjacent saccades of the same polarity for a matched number of saccades divided equally into positive and negative polarities and randomly ordered in time. For each drug, the data shown are from a single representative mouse. Data in $\mathbf{B}$ are presented as the mean \pm standard deviation.

Cocaine, phencyclidine (PCP), and ketamine, an NMDA receptor antagonist that is used as an anesthetic and a recreational drug, induced large numbers of spontaneous eye movements, but only ketamine and phencyclidine impaired rotarod performance. Interestingly, at doses with only a modest effect on the number of visual stimulus-induced $\mathrm{ETM}_{30}$, ethanol induces a distinctive waxing and waning of OKR responsiveness to a continuous visual stimulus with a period of approximately 2-3 minutes (Figure 1B). Similarly, rotarod performance after ethanol administration exhibited large trial-to-trial variability, as reflected in the large standard deviation over multiple trials (Figure 1F).

The survey in Figure 1 does not capture the full range of effects that some of the tested compounds have on spontaneous and/or visual stimulus-induced eye movements, as this experiment reveals only part of a complex time- and dose-dependent response. For example, in earlier experiments with ketamine, we observed time- dependent changes in the slope of the slow component of the OKR and the regularity of stimulus-induced eye movements as well as complete suppression of the OKR during a 5- to 10-minute period after i.p. drug administration (19). Additionally, ketamine induces spontaneous eye movements at low doses but abolishes eye movements at higher doses.

In this study, we have not explored the effects of chronic drug administration on eye movements, an important area for future research given the well-known delay in clinical response seen with many antidepressants and the delayed side effects that accompany chronic treatment with many antipsychotic drugs. We have also made no attempts to distinguish effects referable to peripheral targets - for example, direct actions on the cardiovascular system from actions within the CNS. For most of the tested compounds, abundant evidence implicates CNS targets as their principal sites of action, although in several cases (e.g., cocaine) peripheral targets 
A

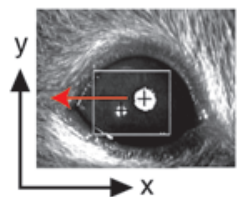

Pre-memantine

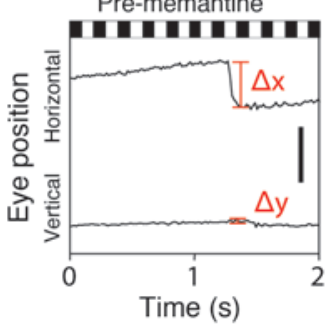

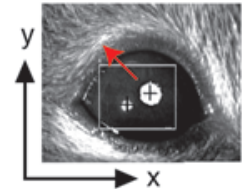

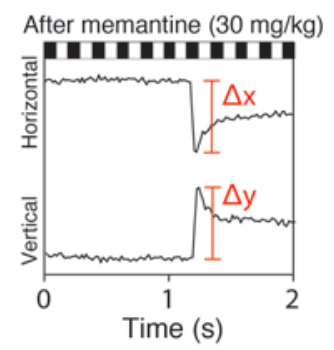

C

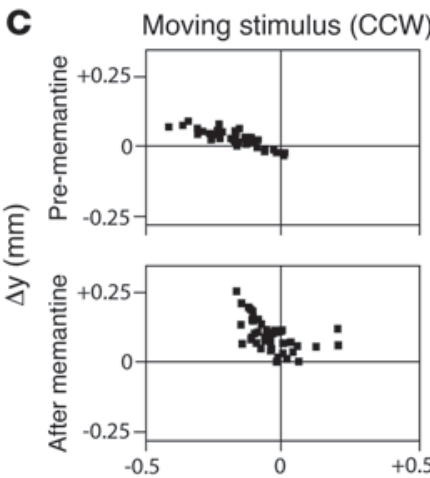

Moving stimulus (CW)
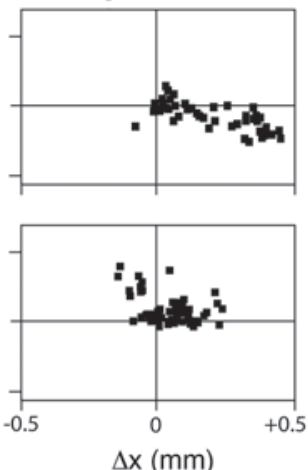

B
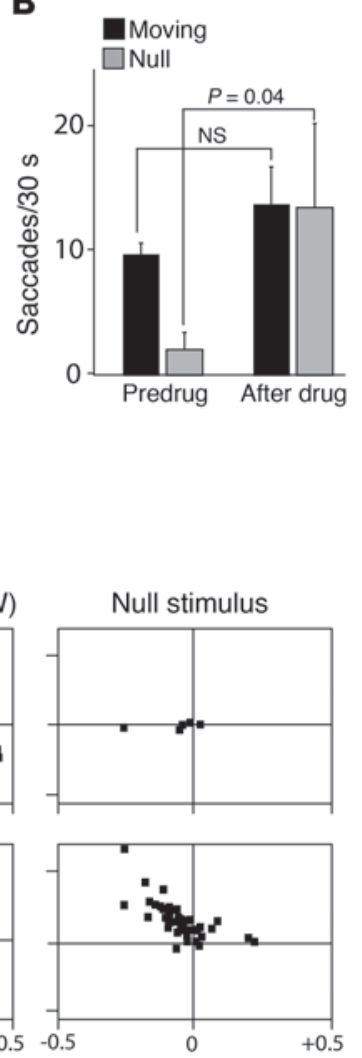

\section{Figure 4}

Memantine induces saccades with a large vertical component. (A) Representative eye movements in response to rotating black and white stripes before or after $30 \mathrm{mg} / \mathrm{kg}$ i.p. memantine. Predrug saccades are confined largely to the horizontal $(x)$ axis, whereas postmemantine saccades also have a large vertical $(y)$ component. Scale bar: $0.5 \mathrm{~mm}$. (B) The number of saccades during alternating 30 -second moving visual stimulus and rest (null stimulus) periods, as defined in Figure 1. Memantine induces spontaneous saccades during the rest periods but has little effect on saccade frequency in response to a visual stimulus. (C) Saccade amplitudes and 2-dimensional trajectories (as defined in $\mathbf{A}$ ) before and after memantine in response to clockwise (CW) or counterclockwise (CCW) rotating visual stimuli or in the absence of a moving stimulus (null). After memantine injection, large vertical components are common in response to CCW motion and among spontaneous eye movements. Data in B are averages from $\mathbf{3}$ mice; data in $\mathbf{C}$ are from a single representative mouse. may also be relevant. Despite its limitations, this survey indicates that the action of a broad subset of psychoactive drugs can be monitored via their effects on eye movements. In the paragraphs that follow, we describe experiments based on acute drug administration to examine dosing, pharmacokinetics, tissue distribution, receptor specificity, and efficacy.

Quantifying dose response and duration of action. Fundamental to investigating drug action in the intact animal is quantification of the dose dependence and time course of the response. In general, the response will be a complex function of pharmacokinetics (drug absorption, distribution, metabolism, and excretion) and pharmacodynamics (the interaction between a drug and its receptor[s] and the dependence of the physiologic response on receptor occupancy). Continuous monitoring of drug action in awake, behaving animals is most readily conducted using simple physiologic measures such as heart rate and blood pressure. By contrast, continuous monitoring of more complex behaviors, such as feeding, drinking, or wheel running, is confounded by temporal variability on short time scales. As described below, the OKR is unusual in that it reflects the function of complex neural circuits, but it can be continuously monitored on time scales of tens of seconds to hours.

To compare the OKR with the rotarod as assays for quantifying the duration of drug action, we monitored the response to phenytoin at 3, 24, and 48 hours after a single i.p. injection of $85 \mathrm{mg} / \mathrm{kg}$ (Figure 2). Consistent with phenytoin's long half-life in humans ( 24 hours), the OKR was eliminated at both 3 and 24 hours after delivery and then returned by 48 hours. Interestingly, at 24 hours after injection, phenytoin induced frequent, small-amplitude, spontaneous eye movements (see insets in the top left panel of Fig- ure 2), reminiscent of the nystagmus that characterizes phenytoin toxicity in humans $(15,16)$. In contrast to the large change in the OKR at 3 and 24 hours after phenytoin injection, rotarod testing showed only a small performance decrement in response to phenytoin at 3 hours after drug delivery.

To assess dose-response relationships, we quantified the dosedependent elimination of the OKR by gabapentin and by 2 benzodiazepines, diazepam and zolpidem (Figure 2). For all 3 drugs we observed a simple dose-dependent reduction in $\mathrm{ETM}_{30}$. The rotarod test showed no significant changes at any of the gabapentin concentrations used, and it was less sensitive than the OKR in detecting the effects of the benzodiazepines. These experiments show that, for this set of 4 drugs, the OKR provides a measure of drug action that is at least as sensitive as rotarod performance.

Drug-induced oculomotor movements not driven by visual stimuli. As described above and shown in Figure 1, A and D, several drugs induce eye movements independent of the moving visual stimulus, referred to hereafter as spontaneous eye movements. In the case of cocaine and phencyclidine, spontaneous eye movements may represent the rodent correlate of the hyper-arousal (cocaine) or delirium/hallucinations (phencyclidine) experienced by humans. To systematically explore parameters associated with spontaneous drug-induced eye movements, we have compared continuous recordings obtained in the absence of a moving visual stimulus, before and after exposure to ketamine, memantine, and phencyclidine (Figure 3). Spontaneous eye movements in control mice typically shift the eye to a new and relatively stable position, generating a distinctive "box-car" shape (Figure $3 \mathrm{~A}$ ). By contrast, the spontaneous eye movements induced by the 3 drugs shown in Fig- 
A
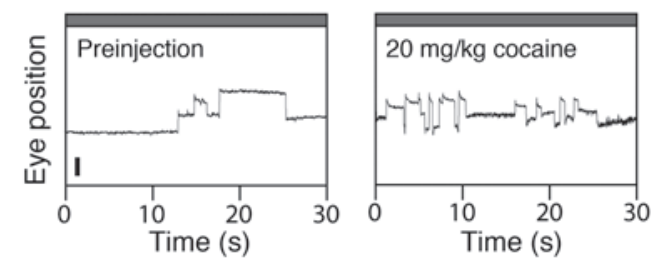

\section{B}
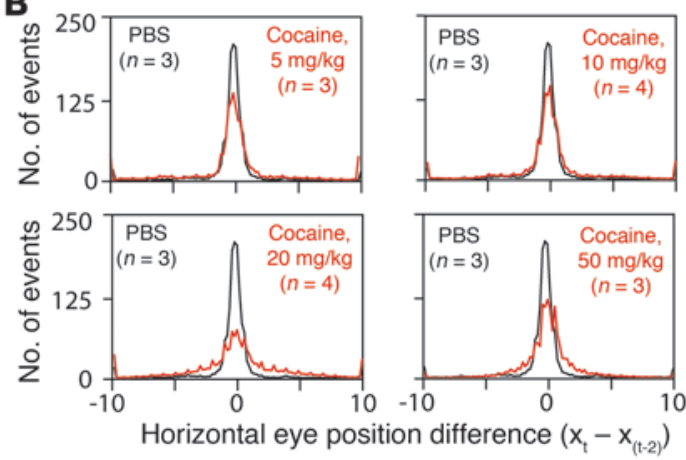

(image units, bin size $=0.2$ )
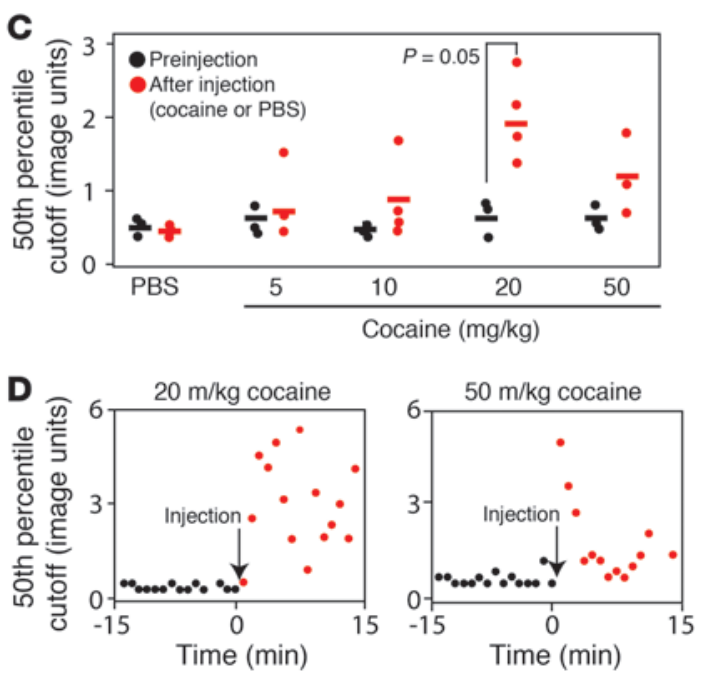

ure 3 more closely resemble a bone fide ETM in that the rapid component is followed by a slower return to baseline; this postsaccade trajectory is reminiscent of the aberrant trajectories of fixational eye movements in primates in the absence of the neural integrator (20) and is quantified for memantine in Figure 3B. For simplicity, we will refer to these drug-induced eye movements as "OKR like" although the slow phase is substantially less linear than the slow phase of true OKR movements.

We have used a series of simple algorithms to analyze the frequency, polarity, and shape of spontaneous eye movements. In brief, the first derivative of eye position with respect to time was calculated, and, then, proceeding sequentially from the start of the record, each contiguous set of first derivative values greater than an empirically determined threshold was counted as a single saccade, and its polarity (nasal or temporal) was recorded. To insure that the rapid rebound associated with OKR-like movements was not counted as a saccade in the opposite direction, the last time point in each set of contiguous suprathreshold first-derivative points was designated as the time of the saccade, and a second saccade designation was not permitted during the following 48

\section{Figure 5}

Quantifying the increase in spontaneous eye movements induced by cocaine. (A) Representative 30-second records of spontaneous eye movements (i.e., with no moving visual stimulus) recorded prior to and after an i.p. injection of $20 \mathrm{mg} / \mathrm{kg}$ cocaine. The box-car shape of the eye movements is retained, but the frequency of movements increases after cocaine administration. (B) Histograms for individual mice showing horizontal eye position differences between time points separated by 2 seconds $\left(X_{t}-X_{t-2}\right)$ and sampled at 16.7-millisecond intervals. Data are the $X_{t}-X_{t-2}$ averages for the 15 minutes immediately after i.p. injection of PBS (black) or cocaine (red) at the indicated doses. $n$ indicates the number of mice averaged per condition. Horizontal eye position $(x)$ is plotted in "image units," a distances measure in the video image. (C) 50th percentile cutoff value for the $X_{t}-X_{t-2}$ distributions for each mouse. Horizontal bars represent the mean. Individual symbols represent individual mice. (D) Time course of spontaneous eye movements plotted for each 30 -second recording interval; the interleaved 30-second intervals with moving black and white stripes were omitted from the analysis. Spontaneous eye movements are quantified as in C. Individual symbols represent individual mice. Data are presented as the mean \pm standard deviation.

milliseconds. To distinguish box-car from OKR-like movements, the average value of the first derivative during the 116 milliseconds after each saccade was calculated; values of less than $0.5-0.8$ $\mathrm{mm} / \mathrm{s}$ were designated as box-car movements, and values greater than $0.5-0.8 \mathrm{~mm} / \mathrm{s}$ were designated as OKR-like movements, with the exact cutoff determined individually for each OKR record. Figure 3C shows the designations of box-car or OKR-like movements for a pair of recordings in the absence of a moving visual stimulus, prior to (Figure 3C, top) and after (Figure 3C, bottom) phencyclidine administration.

The number of spontaneous OKR-like eye movements was dramatically increased by ketamine, memantine, and phencyclidine treatment (Figure 3D). Additionally, for memantine and phencyclidine, spontaneous saccades often occurred in rapid succession, with approximately 0.5 seconds between adjacent saccades (Figure 3E), a pattern reminiscent of a burst of action potentials. Visual inspection further suggests that drug-induced OKR-like movements tend to occur in clusters of the same polarity (Figure 3C). While this phenomenon can also be seen in Figure 1 during the 30-second rest periods in the baclofen, cocaine, ketamine, and phencyclidine records, it is possible that, in the experiments in Figure 1 , the interleaved periods of moving visual stimuli could have entrained the polarity of the spontaneous OKR-like movements. Figure $3 \mathrm{~F}$ shows quantification of the clustering of saccade polarities during an extended recording in the absence of a moving visual stimulus (to eliminate the possibility of entrainment of the spontaneous eye movements) and compares it with the degree of clustering calculated for a randomly ordered series of saccades with a 1:1 ratio of polarities. This analysis indicates that for memantine and phencyclidine, but not for ketamine, adjacent drug-induced OKR-like movements are of the same polarity far more often than would be expected by chance. Thus, a distinct subset of psychoactive drugs can induce clusters of saccades of identical polarity and relatively uniform spacing. Presumably, these epochs reflect distinct states of brain activity.

In the presence of a horizontally moving visual stimulus, as used here, the amplitude of vertical eye movements is typically small. Therefore, in the preceding analyses we have been exclusively concerned with eye movements in the horizontal plane. However, an 
A

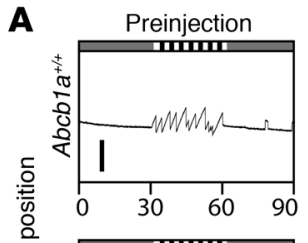
$<1 \mathrm{~h}$ after ivermectin
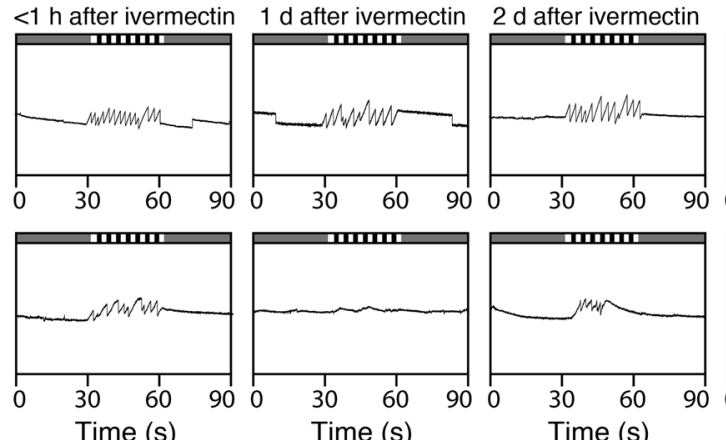

Time (s)
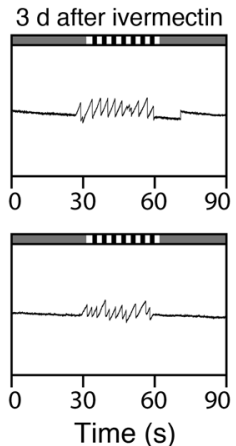

\section{Figure 6}

BBB function assessed by OKR. (A) Time course of the OKR response after i.p. injection of $0.5 \mathrm{mg} / \mathrm{kg}$ i.p. ivermectin in $\mathrm{Abcb} \mathrm{a}^{+/+}$and $\mathrm{Abcb} 1 \mathrm{a}^{-/-}$ littermates. (B and C) Quantification of $(\mathbf{B})$ OKR responses and $(\mathbf{C})$ rotarod performance at the indicated times after $0.5 \mathrm{mg} / \mathrm{kg}$ i.p. ivermectin in

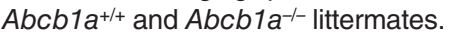
Data in $\mathbf{B}$ and $\mathbf{C}$ are averages from $4 \mathrm{Abcb}_{1 \mathrm{a}^{+/+}}$and $8 \mathrm{Abcb} 1 \mathrm{a}^{-/-}$mice. Scale bar: $1 \mathrm{~mm}$. Data are presented as the mean \pm standard deviation.
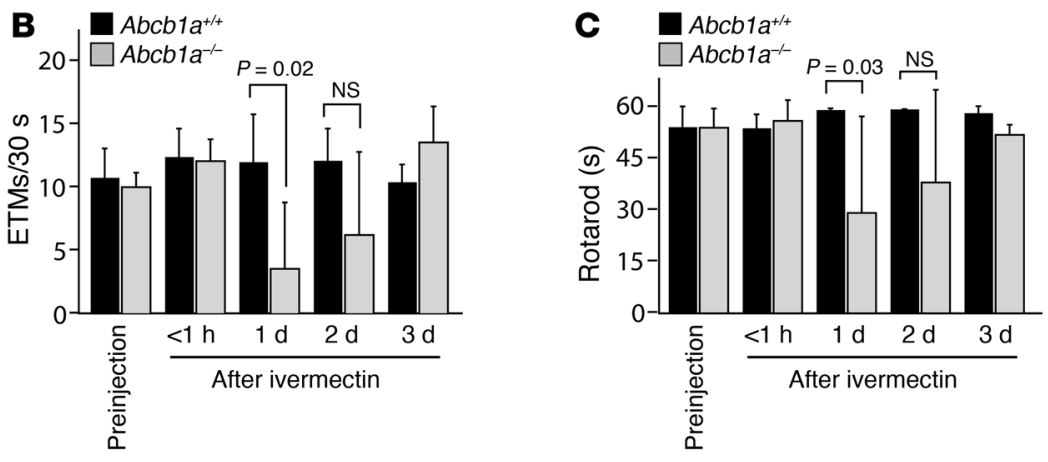

exception to this general pattern was noted after memantine treatment (Figure 4). Both during exposure to the standard horizontally moving visual stimulus and during the interleaved rest period, OKR and OKR-like movements were tilted on an approximately 45-degree angle relative to the horizontal plane, as seen by the roughly equal magnitudes of their horizontal and vertical components. This effect was only observed with counterclockwise visual motion and with spontaneous OKR-like events that mimicked the response to counterclockwise motion. Among the drugs tested thus far, memantine is the only one that shows a substantial shift in the angle of OKR or OKR-like movements. In humans, up-beat nystagmus has been associated with lesions of the cerebellum or medulla, multiple sclerosis, and organophosphate poisoning (11).

Quantifying the effects of cocaine. Cocaine is of special interest, both because of its enormous societal impact and because it affects eye movements in a complex and distinctive manner. As seen in Figure $1 \mathrm{~A}$, at high doses $(50 \mathrm{mg} / \mathrm{kg})$, cocaine abolishes the visual stimulus-induced OKR while simultaneously inducing stereotyped spontaneous OKR-like movements. At lower doses (e.g., 20 $\mathrm{mg} / \mathrm{kg}$ ), cocaine increases the frequency of spontaneous eye movements in the absence of a moving stimulus, with little or no effect on the OKR (Figure 5A and data not shown). Compared with a protocol in which eye movements were monitored in a continuous recording session with no moving stimuli, if the 30 -second periods with no moving stimuli were interleaved between 30 -second periods of rotating black and white stripes (i.e., the standard OKR protocol described in Figure 1A), then the cocaine-induced increase in eye movements was substantially greater. This observation suggests that the hyperactivity of the oculomotor system induced by low-dose cocaine is triggered or gated by recent sensory input. In light of this observation, the experiments shown in Figure 5 were performed with the standard OKR protocol of interleaved stimulus and rest periods.
We have used a simple algorithm to quantify spontaneous eye movements: the horizontal eye position at each time point $\left(X_{t}\right)$ (sampled at $60 \mathrm{~Hz}$ ) was subtracted from the horizontal eye position 2 seconds earlier $\left(X_{t}-X_{t-2}\right)$. For each 30 -second interval, this calculation was performed over the central 26 seconds, and the resulting values were averaged for the 15 preinjection and 15 postinjection 30-second recording intervals. The resulting histograms of $X_{t}-X_{t-2}$ values (averaged from 3 to 4 mice) for PBS or 5, 10, 20 , or $50 \mathrm{mg} / \mathrm{kg}$ cocaine provide a measure of spontaneous activity (Figure 5B). To reduce this measure of spontaneous activity to a single number for each experimental condition, we calculated for each histogram the $X_{t}-X_{t-2}$ value that corresponds to the 50thpercentile cutoff of the distribution (Figure 5C). Interestingly, while Figure 5, A-C, demonstrates a clear increase in spontaneous eye movements at $20 \mathrm{mg} / \mathrm{kg}$ cocaine, spontaneous movements increased to a lesser extent with $50 \mathrm{mg} / \mathrm{kg}$ cocaine. By monitoring individual 30-second intervals, we found that at $50 \mathrm{mg} / \mathrm{kg}$ there is a rapid onset of cocaine-induced spontaneous eye movements, followed within minutes by a decline that represents a general suppression of eye movements (Figure 5D). This analysis establishes a rapid and quantitative method for observing cocaine-induced changes in CNS function.

Quantification of BBB function. One of the most important aspects of preclinical drug development is assessing BBB penetration (5). BBB function is not readily modeled in vitro, as it depends on a complex combination of diffusion barriers and active extrusion by endothelial pumps. From the data presented above, it is clear that the OKR can be used to quantify the CNS actions of a variety of drugs and thereby indirectly measure their penetration across the BBB. To directly address BBB function using the OKR, we compared the responses of WT and $A b c b 1 a^{-/-}$littermates to a single i.p. injection of ivermectin, an antihelmintic drug that activates invertebrate glutamate-gated chloride channels and, at lower efficiency, 
A
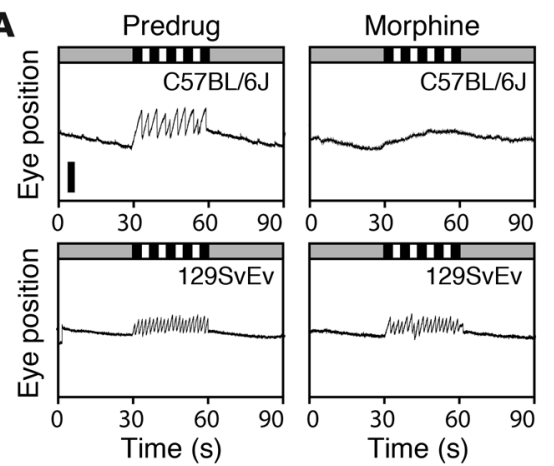

B
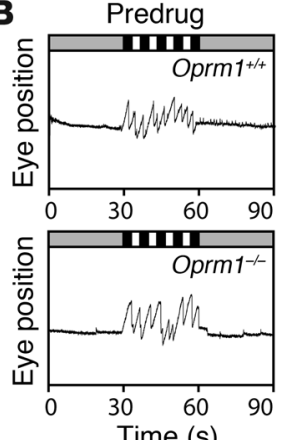

Time (s)
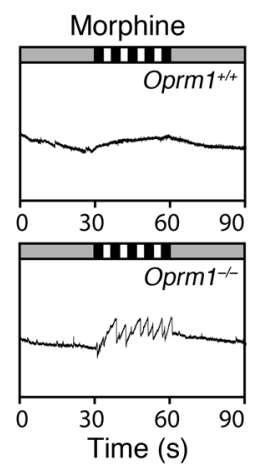

C
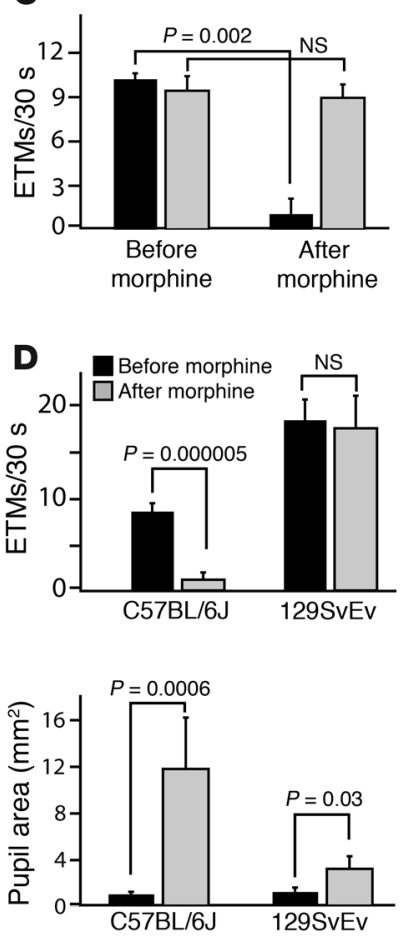
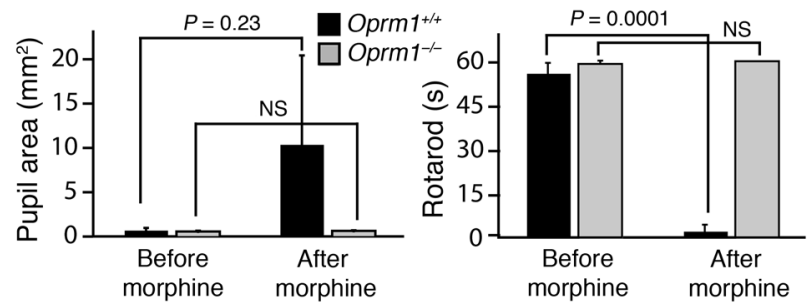

E

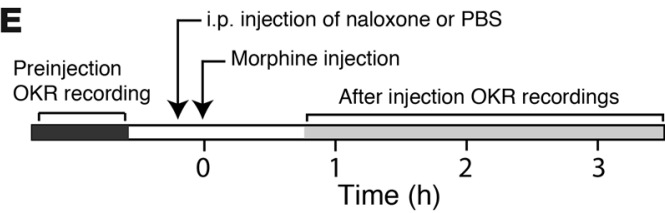

Naloxone + morphine
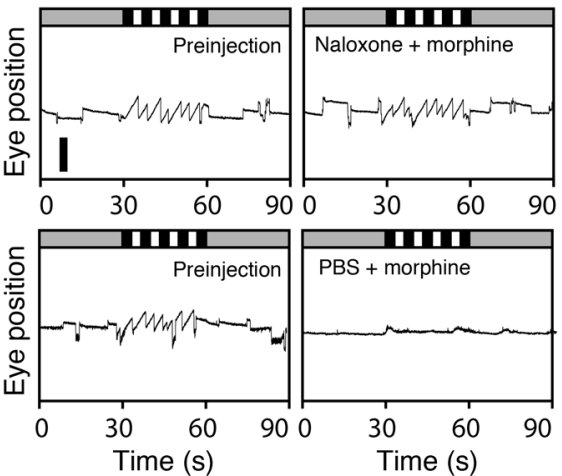

$\square$ PBS + morphine
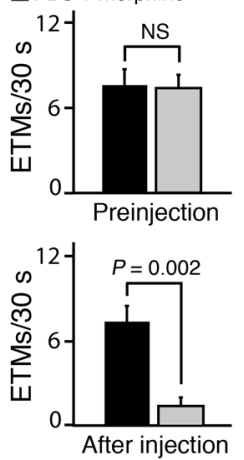

\section{Figure 7}

Strain differences, receptor specificity, and agonist-antagonist interactions analyzed by eye-movement analysis. (A) Morphine $(200 \mathrm{mg} / \mathrm{kg}$, oral delivery) eliminates the OKR in C57BL/6J mice but not in 129SvEv mice. Scale bar: $0.5 \mathrm{~mm}$. (B) OKR suppression by $200 \mathrm{mg} / \mathrm{kg}$ morphine is eliminated in Oprm $1^{-/}$mice but not Oprm1+/+ littermates in a C57BL/6J background. (C) Quantification of the effects of $200 \mathrm{mg} / \mathrm{kg}$ morphine on the OKR, pupil dilation, and rotarod per-

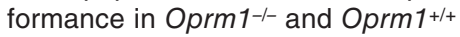
littermates. (D) Quantification of OKR suppression and pupil dilation in C57BL/6J and 129SvEv mice in response to $200 \mathrm{mg} / \mathrm{kg}$ morphine. (E) Naloxone blockade of morphineinduced suppression of the OKR. Time line of drug administration and OKR recordings (top). Representative OKR traces (bottom left). Quantification of $\mathrm{ETM}_{30}$ (bottom right). i.p. naloxone administered at $10 \mathrm{mg} / \mathrm{kg}$ 10 minutes before $200 \mathrm{mg} / \mathrm{kg}$ oral morphine blocked morphine-induced OKR suppression. Data in $\mathbf{C}$ and $\mathbf{E}$ are averages from 3 Oprm1-/- and Oprm1 $1^{+/+}$mice. Data in $\mathbf{D}$ are averages from 6 C57BL/6J and 4 129SvEv mice. Scale bar: $1 \mathrm{~mm}$. Data are presented as the mean \pm standard deviation. vertebrate GABA receptors $(21,22)$. Abcb1a (also known as Mdrla), a broad specificity $\mathrm{ABC}$ transporter that is expressed in endothelial cells within the CNS vasculature, plays a major role in protecting the CNS from ivermectin toxicity (23). As shown in Figure 6, A and $\mathrm{B}$, in $\mathrm{Abcb1a^{-/- }}$ mice, but not in $\mathrm{Abcb1a^{+/+ }}$ littermate controls, the OKR is eliminated 1 day after i.p. injection of $0.5 \mathrm{mg} / \mathrm{kg}$ ivermectin, with a slow recovery over the ensuing 1 to 2 days. The rotarod test shows a similar time course of impaired performance, although the magnitude of the performance decrement is somewhat less than it is for the OKR (Figure 6C). A Kaplan-Meier survival curve for this experiment is shown in Supplemental Figure 2 and confirms the sensitivity of $A b c b 1 a^{-/-}$mice to ivermectin at this dose. Together with the responses to other CNS-active compounds (e.g., Figures 1 and 2), these data indicate that the OKR can be used to quantitatively assess BBB integrity and function.

Receptor specificity and drug-drug interactions. To assess the use of eye-movement testing in the analysis of drug-receptor specific- ity, we compared the effect of orally administered morphine in mice with a targeted deletion of the mu opioid receptor 1 gene (Oprm $1^{-/-}$mice) relative to that in Oprm $1^{+/+}$littermates. Consistent with the known specificity of morphine for mu opioid receptors, Oprm1 $1^{-1-}$ mice appeared to be completely resistant to the effects of morphine, whereas Oprm $1^{+/+}$mice exhibited pupil dilation, suppression of the OKR, and severely degraded rotarod performance (Figure 7, B and C).

Drug-drug interactions can enhance or diminish efficacy by changing the kinetics of drug transformation, metabolism, or excretion or by perturbing intersecting physiologic pathways or different steps in the same pathway. The most direct drug-drug interactions arise from competition for the same receptor binding site(s). Drug-drug interactions can lead to therapeutically advantageous synergies or undesirable side effects and can alter optimal dosing if 2 or more drugs are used simultaneously. To explore the use of the OKR for quantifying drug-drug interactions, we have chosen 
A

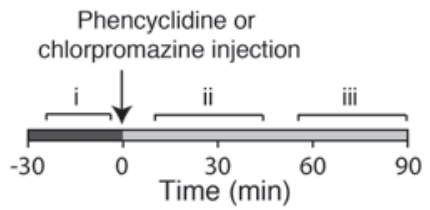

Chlorpromazine Phencyclidine

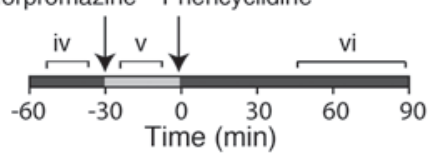

B
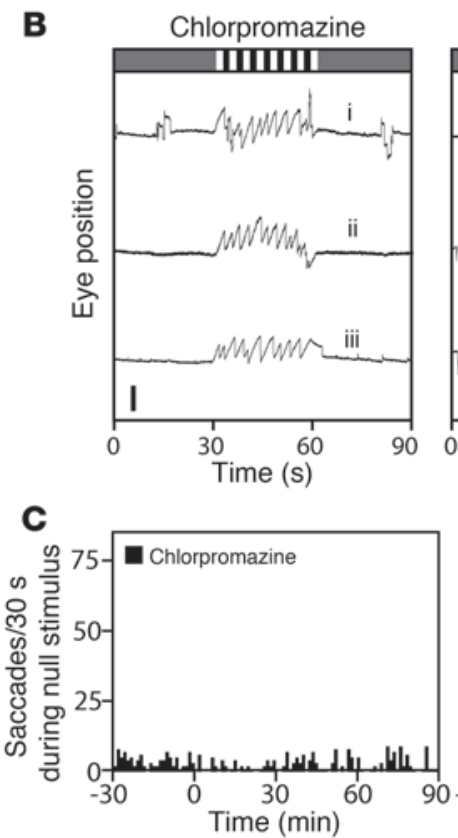

D

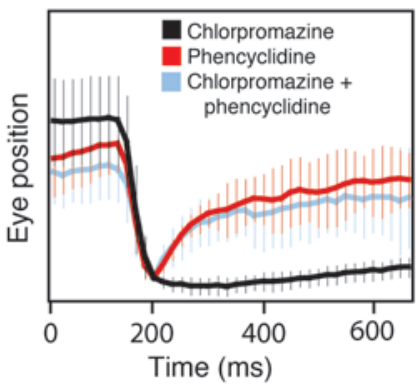

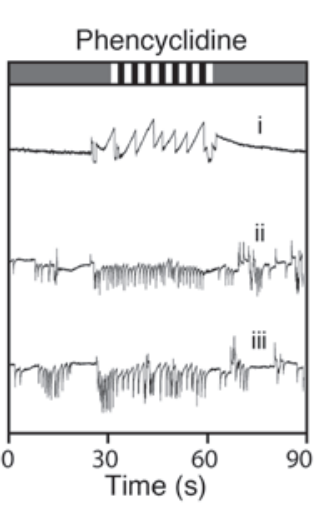

\section{Chlorpromazine} + phencyclidine

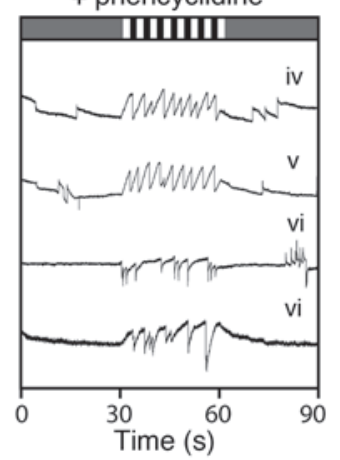

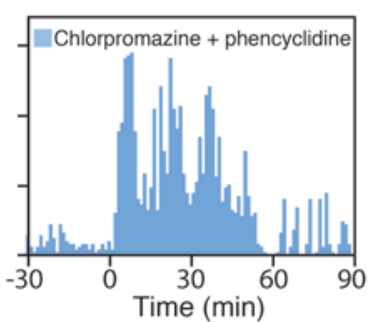

E

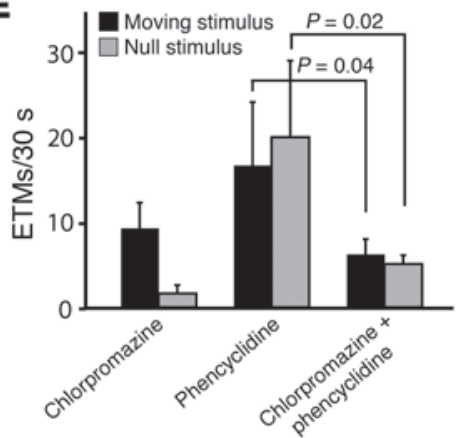

\section{Figure 8}

Antipsychotic drug treatment model monitored by eye-movement analysis. (A) Effect of chlorpromazine $(0.5 \mathrm{mg} / \mathrm{kg})$ and phencyclidine $(10 \mathrm{mg} / \mathrm{kg})$ on the OKR between 0 and 90 minutes after i.p. delivery. Time lines show drug injection times and OKR recording intervals for individual drug experiments (left) and combined drug experiments (right). Segments "i-vi" correspond with traces in B. (B) Beginning within 15 minutes of administration, 10 $\mathrm{mg} / \mathrm{kg}$ phencyclidine produces rapid OKRlike movements independent of the visual stimulus; $0.5 \mathrm{mg} / \mathrm{kg}$ chlorpromazine has little or no effect on the OKR. Beginning approximately 60 minutes after phencyclidine injection, chlorpromazine preinjection substantially suppresses phencyclidineinduced spontaneous OKR-like movements (traces labeled "vi"). Scale bar: 0.5 $\mathrm{mm}$. (C) Saccades per 30 seconds measured during the 30 -second rest intervals during representative 2-hour recordings. (D) Spontaneous eye-movement traces, normalized to the saccade amplitude, for the indicated drug treatments $(n>14$ saccades averaged per condition). (E) Number of saccades per 30-second interval in the presence or absence of a moving visual stimulus for the indicated drug treatments, recorded 45-90 minutes after phencyclidine administration. Data in $\mathbf{E}$ show averages from 3 mice per drug treatment. Data are presented as the mean \pm standard deviation. a classic example of a competitive drug interaction, the blockade of morphine-induced somnolence by the opiate antagonist naloxone. Figure 7E shows the time course of the experiments: the OKR was recorded prior to drug administration, naloxone $(10 \mathrm{mg} / \mathrm{kg})$ or PBS was injected i.p., 15 minutes later morphine $(200 \mathrm{mg} / \mathrm{kg})$ was delivered orally, and then, beginning 45 minutes later, the OKR was recorded for 250 minutes. The preinjection panels in Figure 7E show the expected visual stimulus-induced OKR as well as several box-car eye movements during the rest periods. Morphine treatment paired with a PBS preinjection suppressed both types of eye movements. By contrast, naloxone preinjection completely eliminated the effect of oral morphine. These data indicate that the OKR can be used to quantify the interaction between opiate agonists and antagonists. It could presumably be used in an analogous manner to monitor other classes of drug-drug interactions.

Variations in drug responses have been extensively documented across and within species, and they represent one of the challenges in extrapolating efficacy data from animals to humans. Among inbred lines of mice, there is wide variation in behaviorally measured opiate responses, as seen, for example, in the high-morphine sensitivity of C57BL/6J mice and the low-morphine sensitivity of $129 \mathrm{SvEv}$ mice in locomotion and thermal analgesia tests and in the jumping response to naloxone-induced acute opiate withdrawal (24). Figure 7, A and D, extends these behavioral observations by showing that 129SvEv mice exhibited an unaltered OKR and reduced pupil dilation at a morphine dose $(200 \mathrm{mg} / \mathrm{kg})$ that completely suppresses the OKR and dilates the pupil in C57BL/6J mice.

Quantification of drug efficacy in a schizophrenia model. In humans, chronic phencyclidine or amphetamine abuse can lead to schizophrenia-like symptoms, suggesting that hypoactivity of NMDA signaling (phencyclidine) and/or hyperactivity of serotonin/ dopamine signaling (amphetamine) are relevant to the etiology of schizophrenia $(25,26)$. In rodents, pharmacologic models of schizophrenia have been developed using phencyclidine, ketamine, 

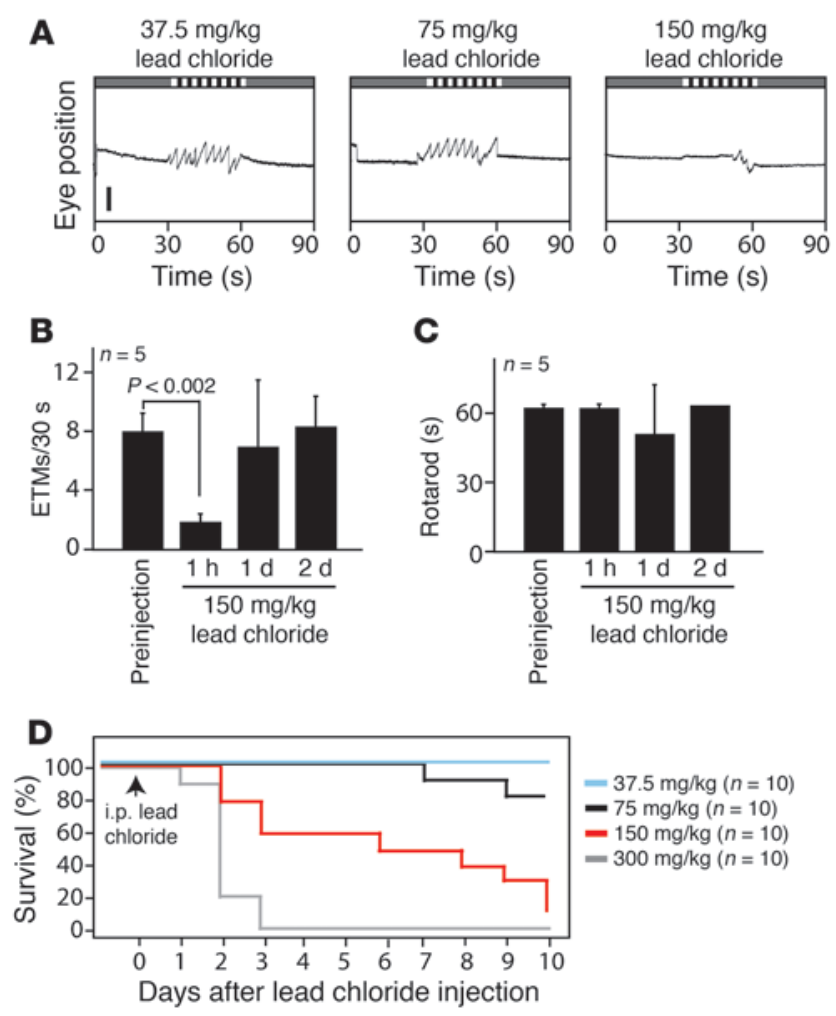

\section{Figure 9}

Acute lead toxicity inhibits the OKR. (A) Representative 90-second OKR records 1 hour after the indicate dose of lead chloride delivered i.p. Five mice were tested at each dose. Scale bar: $0.5 \mathrm{~mm}$. (B and $\mathbf{C}$ ) Quantification of (B) $\mathrm{ETM}_{30}$ and (C) rotarod performance at different times before and after a single injection of $150 \mathrm{mg} / \mathrm{kg}$ lead chloride ( $n=5$ mice). (D) Kaplan-Meier survival curve for a single i.p. dose of lead chloride at the indicated doses ( $n=10$ mice per group). Data are presented as the mean \pm standard deviation.

or amphetamine treatment (27). The large literature on eye-tracking defects in schizophrenics (e.g., ref. 28) and the observation that many antipsychotic drugs affect eye movements in both healthy volunteers and schizophrenics $(14,29)$ suggest that changes in eye movements could be used to quantify disease severity or treatment efficacy in animal models of schizophrenia.

As described above, phencyclidine treatment in mice induced highfrequency spontaneous OKR-like eye movements (Figure 1D and Figure 3), an effect that lasted for at least 90 minutes after an i.p. injection of $5 \mathrm{mg} / \mathrm{kg}$ (Figure $8, \mathrm{~A}$ and B). Chlorpromazine at $0.5 \mathrm{mg} / \mathrm{kg}$ had little effect on the OKR (Figure 8, A and B), although at $5 \mathrm{mg} / \mathrm{kg}$ it substantially slowed the response (Figure 1, A and C). Interestingly, if chlorpromazine $(0.5 \mathrm{mg} / \mathrm{kg})$ was administered 30 minutes before phencyclidine $(10 \mathrm{mg} / \mathrm{kg})$, spontaneous eye movements were substantially suppressed and the OKR was reduced from approximately $15 \mathrm{ETM}_{30}$ with phencyclidine alone to approximately $6 \mathrm{ETM}_{30}$ with both phencyclidine and chlorpromazine, although in the latter case the slope of the slow components was steeper than that in control ETMs (Figure 8, B-E). This chlorpromazine-mediated suppression began approximately 45 minutes after phencyclidine administration, and it persisted for at least 1 hour. These observations suggest that the suppression of phencyclidine-induced spontaneous eye movements could be used as a screening tool for antipsychotic drug candidates.
Application to toxicology. Assessing the effects of exposure to environmental chemicals, either natural or man-made, represents a major public health endeavor. Heavy metals - in particular, lead, cadmium, mercury, and arsenic - have long been known to impair a wide variety of physiologic functions after acute or chronic exposure. Although lead exposure has declined worldwide over the past several decades, it remains a major public health risk due to its widespread industrial use, especially in the developing world (30). The neurologic sequelae of lead exposure ("lead encephalopathy") have been extensively studied. While several studies have examined the effects of chronic lead exposure on eye movements in humans and laboratory animals (31-33), to our knowledge there are no reports on eye-movement effects in the context of acute lead toxicity.

To examine the effects of acute lead toxicity, adult mice were given a single i.p. injection of $37.5,75,150$, or $300 \mathrm{mg} / \mathrm{kg}$ lead chloride $(n=10$ mice for each group). The resulting Kaplan-Meier survival curves show a delayed and dose-dependent lethality after lead exposure, with a dose of $37.5 \mathrm{mg} / \mathrm{kg}$ showing no lethality over the ensuing 10 days and a dose of $300 \mathrm{mg} / \mathrm{kg}$ producing death within 1-3 days (Figure 9D). When ETMs were monitored 1 hour after injection, there was little or no effect of exposure at doses of 37.5 and $75 \mathrm{mg} / \mathrm{kg}$, but a nearly complete loss of eye movements at doses of 150 and $300 \mathrm{mg} / \mathrm{kg}$ (Figure 9, A and B, and data not shown). For the $150 \mathrm{mg} / \mathrm{kg}$ dose, eye movements were monitored over the ensuing 2 days and showed a full recovery to baseline (Figure 9B). When rotarod performance was monitored in the same mice, there was little or no effect of $150 \mathrm{mg} / \mathrm{kg}$ lead at any of these time points (Figure 9C). The rapid loss and subsequent recovery of the OKR after the administration of $150 \mathrm{mg} / \mathrm{kg}$ lead implies an acute toxic process that is operationally distinguishable from the progressive loss of viability that occurred over the following 2-10 days (Figure 9D). Chronic toxicity likely involves the direct inhibition by lead of a variety of enzymes as well as competition by lead for metal binding sites during the biosynthesis of metalloproteins (34). It is an open question whether the rapid and transient lead inhibition of the OKR can be explained by either of these mechanisms. These data support the general idea that monitoring eye movements might be a broadly useful method for assessing chemical toxicities.

Eye movements in a Huntington disease model. The use of eye-movement testing would be substantially enhanced if it could be used not only to monitor drug responses in otherwise normal mice but also drug efficacy in the treatment of CNS disease. In humans, many degenerative CNS diseases are associated with aberrant eye movements (11). For example, patients with Huntington disease (HD) exhibit errors in saccade timing, direction, and accuracy during fixation tasks $(35,36)$, and presymptomatic carriers of an HD mutation produce more errors than controls on a variety of saccade tasks (37). Thus, eye-movement abnormalities represent a potential biomarker for HD.

In the mouse, overexpression of an $\mathrm{N}$-terminal Huntingtin protein fragment with an expanded polyglutamine repeat from the $\mathrm{Htt}$ $R 6 / 2$ transgene produces a progressive neurodegeneration associated with distinctive motor symptoms and transcriptional changes characteristic of $\mathrm{HD}$ in humans $(38,39)$. $H t t R 6 / 2$ heterozygotes are typically asymptomatic before approximately 9-11 weeks of age and then show a rapid decline, with death occurring at approximately 10-13 weeks. To explore the use of eye-movement analysis for assessing disease progression in $H t t R 6 / 2$ mice, transgenic and nontransgenic female littermates were tested, beginning at 8 weeks of age, when rotarod performance, appearance, and gener- 

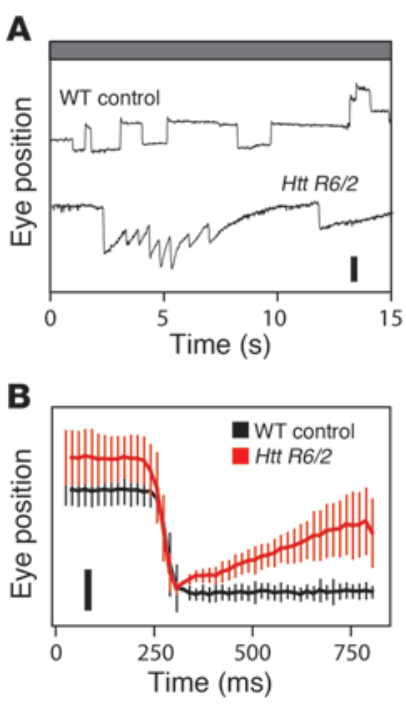

C

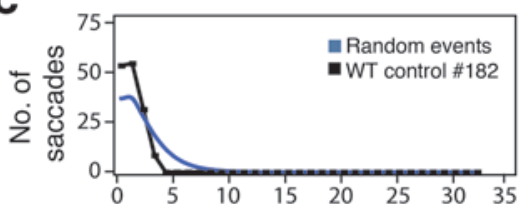

흄
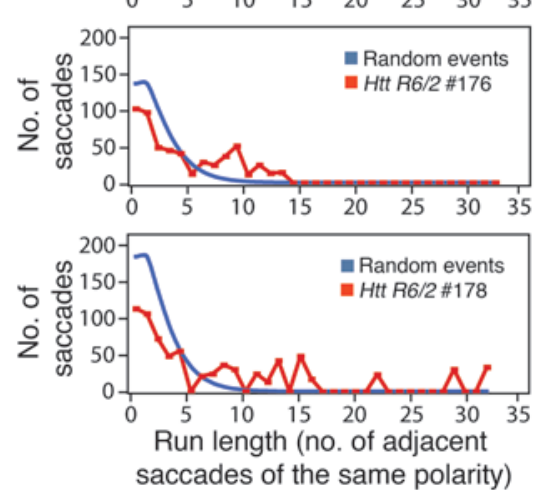

\section{Figure 10}

Spontaneous eye movements in a mouse model of HD. (A) $H t t$ R6/2 transgenic mice have spontaneous OKR-like eye movements at 8 to 12 weeks of age. Scale bar: $0.5 \mathrm{~mm}$. (B) Spontaneous eye-movement traces, normalized to saccade amplitude, for the mice shown in $\mathbf{A}$ ( $n=10$ saccades for WT control and 36-40 saccades for $\mathrm{Htt} R 6 / 2$ ). Scale bar: $0.25 \mathrm{~mm}$. (C) Clustering of saccade polarity. Black (WT) and red (Htt R6/2) lines show the number of adjacent saccades of the same polarity over a 5-minute recording interval. \#182, \#176, and \#178 refer to individual mice. Blue lines show the calculated distributions for the same number of saccades divided equally into positive and negative polarities and randomly ordered in time. Data are presented as the mean \pm standard deviation. al health of control and experimental mice were indistinguishable. Interestingly, at this age, $\mathrm{Htt} \mathrm{R} 6 / 2$ mice showed spontaneous eye movements that resembled those induced by phencyclidine and memantine in their propensity to occur in runs of the same polarity but resembled visually evoked ETMs in the linearity of the slow component (Figure 10). These eye movements may represent the murine correlate of the saccade errors made by HD patients, and they could presumably be used to follow disease progression or responses to treatment in presymptomatic HD animal models.

\section{Discussion}

The experiments described above establish eye-movement analysis as a broadly useful tool for rapidly and quantitatively assessing the responses of mice to a wide variety of psychoactive compounds. The eye movement effects include a slowing or complete suppression of the OKR, a shift in eye-movement direction, induction of spontaneous OKR-like movements in the absence of visual stimuli, clustering of the polarity or timing of OKR-like movements, and changes in the slope of the slow phase of the OKR-like movements. By monitoring these parameters, eye movements can be used to quantify drug dose, duration of action, BBB penetration, receptor specificity, and agonist-antagonist interactions. We further show that the frequency of OKR-like events can be used to monitor (a) the therapeutic effect of antipsychotic drug treatment in a pharmacologic model of schizophrenia and (b) the presence of disease in a mouse model of HD.

Based on the pharmacologic specificity of the drugs tested here, it appears that a wide variety of neurotransmitter systems can influence eye movements in mice, including cholinergic, glutamatergic, aminergic, GABAergic, opioid, and cannabinoid systems. At present, we do not know which drugs directly perturb eye-movement circuits and which act indirectly via changes in arousal, coordination, or higher mental states. In the paragraphs that follow, we relate our findings to the known effects of psychoactive drugs and CNS diseases on eye movements in humans, and we discuss potential applications of eye-movement analysis to drug discovery, drug abuse, and CNS disease research.

Comparison of the effects of psychoactive drugs on mouse and human eye movements. Eccentric gaze, smooth pursuit, and convergent eye movements are easily elicited in humans and are an integral part of the standard neurologic examination (40). Each of these eye movements either brings the object of regard onto the fovea or serves to maintain it there. The rodent retina lacks a specialized region devoted to high acuity vision, and, as a result, rodent eye movements are not driven by foveal mechanisms. However, rodents resemble humans and other primates in possessing reflexes for stabilizing the image on the retina. These reflexes compensate for angular and linear self-motion and are based on vestibular input (VOR) and on movement of the image across the retina (the OKR). As the midbrain and hindbrain nuclei that subserve the VOR and the OKR are highly conserved among mammals, it is reasonable to expect a corresponding evolutionary conservation of neurochemistry and neural circuitry within these systems. Current evidence supports this expectation (41).

Changes in eye movements in response to acute administration of compounds representing the major classes of psychoactive drugs have been extensively studied in humans. Smooth pursuit, convergence, and the maintenance of eccentric gaze are especially sensitive to drug administration (11). If we consider only smooth pursuit eye movements - the human response that most closely resembles the mouse OKR - we observe a high degree of similarity in the effects of a number of drugs between the 2 species (human data are summarized in refs. 11 and 14). Benzodiazepines, barbiturates, opiates, and phenytoin impair smooth pursuit in humans and diminish or eliminate the OKR in mice. In humans, acute administration of haloperidol or chlorpromazine, typical first generation antipsychotic drugs (i.e., antagonists at D2-D4 dopamine receptors), or clozapine, a second generation antipsychotic (i.e., a mixed dopamine and serotonin receptor antagonist), decreases smooth pursuit eye movements. In mice, all 3 antipsychotics produce a marked slowing of the OKR. In humans, cocaine induces opsoclonus (spontaneous large-amplitude saccades) and phencyclidine induces nystagmus (repetitive OKR-like movements). In mice, both drugs induced spontaneous eye movements. In humans, amphetamine has little effect on smooth pursuit or saccadic eye movements, and amphetamine similarly has little or no effect on the mouse OKR.

Other drugs show more or less divergent responses between the 2 species. For example, in humans, carbamazepine (an anticonvul- 
sant and mood-stabilizing drug that potentiates GABA function and decreases sodium channel opening) impairs smooth pursuit eye movements, but, in the present study, it did not affect the mouse OKR. Baclofen, a GABA derivative used in the treatment of spasticity, including periodic alternating nystagmus in humans, paradoxically induces OKR-like eye movements in mice. Nicotine impairs smooth pursuit eye movements in humans but had no apparent effect on the mouse OKR. It is unclear whether these response differences reflect a fundamental divergence between humans and mice in neurochemistry and/or neural circuitry or differences in pharmacokinetics, pharmacodynamics, drug dosing, the sensitivity of eye-movement testing protocols, or some combination of these factors.

Eye-movement defects and CNS disease in humans. In humans, a wide variety of focal CNS lesions, metabolic disorders, and progressive degenerative diseases produce eye-movement defects as one part of a constellation of clinical signs and symptoms. Among the degenerative diseases that typically include eye-movement defects are multiple sclerosis, Parkinson disease, HD, progressive supranuclear palsy, the hereditary cerebellar ataxias, Alzheimer disease, Creutzfeldt-Jacob disease, and AIDS dementia (11). Although mouse models have been developed for many of these diseases, thus far there has been little or no discussion of eye-movement defects. Instead, the small number of studies that have examined eye movements (the OKR and/or the VOR) in genetically modified mice have focused on gene defects that lead to selective retinal, vestibular, or cerebellar dysfunction but do not model the major human neurodegenerative diseases $(17,42-46)$. Our observation of a progressive increase in spontaneous OKR-like movements in a mouse model of HD that begins before overt systemic motor symptoms suggests that monitoring eye movements could represent a useful screening tool for preclinical therapeutic trials in this HD model and that a careful assessment of eye movements is warranted in other mouse models of CNS disease.

Evidence for an association between eye-movement defects and neuropsychiatric disease was first reported by Diefendorf and Dodge (47). In the century since that time, a large number of studies have supported the general concept that abnormalities in smooth pursuit and voluntary saccades represent a biomarker for schizophrenia, although confounding factors, such as previous or current drug treatment and nicotine exposure from cigarette smoking, have not been entirely eliminated (28). We report here that commonly used pharmacologic models of schizophrenia - created by treatment with ketamine or phencyclidine - produce distinctive and readily quantified eye-movement abnormalities in mice. We also observed the abrogation of phencyclidine-induced OKR-like movements after chlorpromazine pretreatment, an interaction that could represent a mouse model of antipsychotic drug action. We note that the mechanism by which this abrogation occurs may be complex, as both drugs have multiple CNS targets: phencyclidine is both an NMDA receptor antagonist (like ketamine) as well as a D2 dopamine receptor agonist, whereas chlorpromazine is an antagonist at dopaminergic, adrenergic, cholinergic, and histaminergic receptors (48).

Potential applications of eye-movement testing in drug discovery. Highthroughput biochemical and cell-based screens with large libraries of compounds are now routine in the pharmaceutical industry. These screens, together with lead optimization, typically generate large numbers of candidate compounds for animal testing. However, animal testing is expensive and time consuming, and, as such, represents a major bottleneck in the drug development pipeline. This limitation is especially acute in CNS drug development, because behavioral parameters are generally more challenging to quantify and interpret than are biochemical, physiological, or histological ones. One strategy to address this bottleneck uses invertebrate model organisms, such as Caenorhabditis elegans and Drosophila melanogaster, for high-throughput animal screening (49, 50). A more promising model is the zebrafish Danio rerio, which is amenable to large-scale continuous monitoring of motor activity $(51,52)$.

As currently practiced, preclinical drug testing focuses almost exclusively on mammals, based on the reasonable assumption that the predictive value of the preclinical test correlates with the evolutionary distance between humans and the animal model. This assumption appears to be especially reasonable in the case of psychoactive drugs, many of which interact with multiple molecular targets, as seen, for example, in the interactions of antidepressant or antipsychotic drugs with multiple classes of amine transporters or receptors, respectively (53). Eye-movement analysis represents a potentially useful addition to the existing repertoire of behaviorally based drug assays. Since it is safe and noninvasive, it could be readily extended to larger mammals, including humans.

Based on the data presented here, the OKR appears to be especially suitable for quantifying sedation, an undesirable side effect of many medications. Thus, for drug development programs aimed at peripheral targets, the OKR could be used as an initial screening tool to identify and eliminate sedating compounds. The OKR could also guide lead optimization efforts aimed at decreasing or increasing BBB penetration for drug development programs with peripheral or central targets, respectively. The distinctive eyemovement responses that mice exhibit to various drugs of abuse - including cocaine, phencyclidine, opiates, ethanol, and cannabinoids - suggest that eye-movement assays might be used to test compounds that counteract the effects of these drugs (54). In the future, it will be interesting to extend these eye-movement studies to include the full range of CNS-active compounds - both therapeutic and toxic - to which humans are exposed.

\section{Methods}

Mouse surgery, $O K R$, and rotarod tests. Methods for surgically inserting a head post, immobilizing the head-posted mouse, presenting a rotating visual stimulus, infrared video recording of eye position, and thresholding and smoothing of the raw eye position data are described in ref. 19. Surgical procedures as well as mouse handling and housing were approved by the Institutional Animal Care and Use Committee of the Johns Hopkins University School of Medicine. Unless otherwise noted, tests were performed with 2- to 7-month-old C57BL/6J female mice, weighing between 18 and 30 grams. The OKR stimulus consisted of alternating vertically oriented black and white stripes, the width of each subtending 4 degrees of visual angle, with the entire pattern, which covered the inside of the testing cylinder, rotating at 5 degrees per second. Head post surgery was conducted at least 48 hours prior to testing to allow adequate time for recovery. Morphine was delivered by i.p. injection (Figure 1) or oral gavage (Figure 7); all other drugs were delivered by i.p. injection. Unless otherwise noted, postdrug OKR recordings were performed between 30 and 90 minutes after drug administration, and the rotarod tests were performed on each mouse immediately after the OKR recording. For rotarod testing, the cylinder rotated at 7 revolutions per minute, and each mouse was given 3-15 sixtysecond trials. Prior to drug injection, each mouse was given up to 3 practice runs on the rotarod. Rotarod data collection began when the mouse attained its first 60 -second rotarod time. 
Pupil area measurements. The area of the pupil was calculated from the infrared video image using ISCAN software.

Drug formulation and delivery. Water soluble compounds were dissolved in PBS or $0.1 \mathrm{M} \mathrm{NaCl}$ for i.p. injection. Hydrophobic compounds were dissolved in a small volume of DMSO or ethanol and then dissolved in Sunflower seed oil (Sigma-Aldrich) (see Supplemental Table 1). For compounds that had been dissolved in ethanol, the ethanol was removed from the ethanol/oil mixture by spinning the sample in a SpeedVac Concentrator (Savant) prior to i.p. injection.

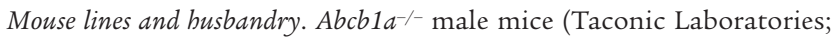
MDR1A-M-M, FVB.129P2-Abcb1 ${ }^{\text {atm1Bor }}$ N7) were crossed to C57BL/6J female mice, and the heterozygous progeny were intercrossed to produce littermate $A b c b 1 a^{+/+}$and $A b c b 1 a^{-/-}$mice for OKR testing. The original $A b c b 1 a^{-/-}$ line from Taconic Laboratories had been backcrossed to the FVB strain, thereby introducing 2 complications for OKR analysis. First, the mice were albino, and iris pigmentation is needed for automated localization of the pupil. Second, the mice were homozygous for the retinal degeneration allele, $P d e 6 b^{r d}$, thus eliminating visually evoked eye movements. Thus, among the F2 littermates, albino and/or Pde6b ${ }^{\text {rd } / r d}$ mice were eliminated from testing. Oprm 1 $1^{-1-}$ male mice (The Jackson Laboratory; stock no. 007559) were crossed to C57BL/6J female mice, and the heterozygous progeny were intercrossed to produce littermate Oprm $1^{+/+}$and $O p r m 1^{-/-}$mice for OKR testing. $H t t$ R6/2 male mice (The Jackson Laboratory; stock no. 002810) were bred to $\mathrm{C} 57 \mathrm{BL} / 6 \mathrm{~J}$ female mice, and heterozygous progeny were tested. $129 \mathrm{SvEv}$ mice were purchased from Taconic (stock no. 129SVE), and C57BL/6J mice were purchased from The Jackson Laboratory (stock no. 000664). PCR primers for genotyping are listed in Supplemental Table 3.

Algorithms for identifying, categorizing, and averaging saccades. Calculations were performed in Microsoft Excel. The fast component of the saccade was identified, and its polarity was scored by thresholding the first derivative of the smoothed eye position record as described in ref. 19. In brief, the time derivative was determined for adjacent eye position data points collected at 16-millisecond intervals, and a threshold for the absolute value of the derivative was set at $1.8-3.6 \mathrm{~mm} / \mathrm{s}$ and used to identify saccades, with the exact threshold determined individually for each OKR record. Box-car and OKR-like saccades were distinguished by determining the first derivative averaged over the first 116 milliseconds after the end of the fast component. Derivatives of less than or greater than $0.5-0.8 \mathrm{~mm} / \mathrm{s}$ were classified as box-car or OKR-like, respectively, with the exact cutoff determined individually for each OKR record. Saccades were averaged by both normalizing the amplitude and aligning the time of the fast component.

Analysis for spontaneous eye movements. For polarity analysis, recordings of 5- or 10-minute duration were obtained in the absence of a moving visual stimulus, saccades were identified as described above, and negative or positive saccade polarities were represented by -1 or +1 , respectively. From the resulting sequence of +1 's and -1 's, the number of runs was tabulated for each possible run length, a run being defined as a series of adjacent saccades of the same polarity. From this analysis, the number of saccades present for each run length was plotted. For comparison with these experimental distributions, we have calculated the distribution of run lengths for a sequence of randomly ordered polarities. Taking the 2 polarities as equally likely, the probability of encountering a run of either polarity and of length $n$ is $2 \times 0.5^{n+2}$, the $n+2$ exponent reflecting the requirement for a saccade of opposite polarity immediately prior to and after the run. Therefore, the probability of a saccade being in a run of length $n$ is proportional to $n \times 2 \times 0.5^{n+2}$.

For the quantification of spontaneous eye movements induced by cocaine, the horizontal eye position at each time point (sampled at $60 \mathrm{~Hz}$ ) was subtracted from the horizontal eye position 2 seconds earlier $\left(X_{t}-X_{t-2}\right)$. For each 30 -second interval, this calculation was performed over the central 26 seconds. To reduce this measure of spontaneous activity to a single number, we calculated for each distribution the $X_{t}-X_{t-2}$ value that corresponds to the 50th-percentile cutoff.

Lead chloride experiments. For the Kaplan-Meier survival curve, cohorts of 10 mice (C57BL/ 6 females, 3-8 months of age), weighing 17-32 grams, were injected i.p. with $37.5,75,150$, or $300 \mathrm{mg} / \mathrm{kg}$ lead chloride in water on day 0 . In addition to the usual ad libitum food and water, an aquagel pack and extra food were placed in the cage. These cohorts and the cohorts used in the OKR and rotarod experiments exhibited very similar dose-dependent survival curves.

Statistics. Student's $t$ test was used for pairwise comparisons. $P$ values less than 0.05 were considered significant. For comparisons of eye movements, a 2 -tailed $t$ test was used. For rotarod comparisons in which the control mice remained on the rotarod for the full 60 seconds, a 1-tailed $t$ test was used.

\section{Acknowledgments}

The authors thank Jay Baraban, Alex Kolodkin, Se-Jin Lee, Randy Reed, King-Wai Yau, and David Zee for advice and/or comments on the manuscript. This work was supported by the Howard Hughes Medical Institute.

Received for publication October 26, 2010, and accepted in revised form June 15, 2011.

Address correspondence to: Jeremy Nathans, 805 PCTB, 725 North Wolfe Street, Johns Hopkins University School of Medicine, Baltimore, Maryland 21205, USA. Phone: 410.955.4679; Fax: 410.614.0827; E-mail: jnathans@jhmi.edu.
1. Kola I, Landis J. Can the pharmaceutical industry reduce attrition rates? Nat Rev Drug Discov. 2004; 3(8):711-715.

2. Pangalos MN, Schechter LE, Hurko O. Drug development for CNS disorders: strategies for balancing risk and reducing attrition. Nat Rev Drug Discov. 2007;6(7):521-532.

3. McDonald K, Trick L, Boyle J. Sedation and antihistamines: an update. Review of inter-drug differences using proportional impairment ratios. Hum Psychopharmacol. 2008;23(7):555-570.

4. Brunner D, Nestler E, Leahy E. In need of highthroughput behavioral systems. Drug Discov Today. 2002;7(18 suppl):S107-S112.

5. Reichel A. The role of blood-brain barrier studies in the pharmaceutical industry. Curr Drug Metab. 2006; 7(2):183-203.

6. Crawley JN. What's Wrong With My Mouse? Behavioral Phenotyping of Transgenic and Knockout Mice. New York, New York, USA: Wiley-Liss; 2000.
7. Tecott LH, Nestler EJ. Neurobehavioral assessment in the information age. Nat Neurosci. 2004;7(5):462-466. 8. de Visser L, van den Bos R, Kuurman WW, Kas MJ, Spruijt BM. Novel approach to the behavioural characterization of inbred mice: automated home cage observations. Genes Brain Behav. 2006;5(6):458-466.

9. Bonasera SJ, Schenk AK, Luxenberg EJ, Tecott LH. A novel method for automatic quantification of psychostimulant-evoked route-tracing stereotypy: application to Mus musculus. Psychopharmacology (Berl). 2008;196(4):591-602.

10. Hall DA, Stanis JJ, Marquez Avila H, Gulley JM. A comparison of amphetamine- and metamphetamine-induced locomotor activity in rats: evidence for qualitative differences in behavior. Psychopharmacology (Berl). 2008;195(4):469-478.

11. Leigh RJ, Zee DS, eds. The Neurology of Eye Move ments. 4th ed. Oxford, United Kingdom: Oxford University Press; 2006.

12. Rothenberg S, Schottenfeld S, Selkoe D, Gross K.
Specific oculomotor deficit after acute methadone. II. Smooth pursuit eye movements. Psychopharmacology (Berl). 1980;67(3):229-234.

13. Rothenberg SJ, Selkoe D. Specific oculomotor deficit after diazepam. II. Smooth pursuit eye movements. Psychopharmacology (Berl). 1981;74(3):237-240.

14. Reilly JL, Lencer R, Bishop JR, Keedy S, Sweeney JA. Pharmacological treatment effects on eye movement control. Brain Cogn. 2008;68(3):415-435.

15. McNamara JO. Drugs effective in the therapy of the epilepsies. In: Hardman JG, Limbird LE, eds. Goodman and Gilman's The Pharmacologic Basis of Therapeutic. 10th ed. New York, New York, USA: McGraw Hill; 2001:521-547.

16. Hogan RE, Collins SD, Reed RC, Remler BF. Neuroophthalmological signs during rapid intravenous administration of phenytoin. J Clin Neurosci. 1999; 6(6):494-497.

17. Stahl JS. Using eye movements to assess brain function in mice. Vision Res. 2004;44(28):3401-3410. 
18. Iwashita M, Kanai R, Funabiki K, Matsuda K, Hirano T. Dynamic properties, interactions and adaptive modifications of vestibulo-ocular reflex and optokinetic response in mice. Neurosci Res. 2001; 39(3):299-311.

19. Cahill H, Nathans J. The optokinetic reflex as a tool for quantitative analyses of nervous system function in mice: application to genetic and druginduced variation. PLoS One. 2008;3(4):e2055.

20. Cannon SC, Robinson DA. Loss of the neural integrator of the oculomotor system from brain stem lesions in monkey. J Neurophysiol. 1987;57(5):1383-1409.

21. Dawson GR, et al. Anticonvulsant and adverse effects of avermectin analogs in mice are mediated through the gamma-aminobutyric acid(A) receptor. J Pharmacol Exp Ther. 2000;295(3):1051-1060.

22. Wolstenholme AJ, Rogers AT. Glutamate-gated chloride channels and the mode of action of the avermectin/milbemycin anthelmintics. Parasitology. 2005;131 suppl:S85-S95.

23. Schinkel AH, et al. Disruption of the mouse mdr1a P-glycoprotein gene leads to a deficiency in the blood-brain barrier and to increased sensitivity to drugs. Cell. 1994;77(4):491-502.

24. Kest B, Palmese CA, Juni A, Chesler EJ, Mogil JS. Mapping of a quantitative trait locus for morphine withdrawal severity. Mamm Genome. 2004;15(8):610-617.

25. Javitt DC. Glutamate and schizophrenia: phencyclidine, N-methyl-D-aspartate receptors, and dopamine-glutamate interactions. Int Rev Neurobiol. 2007;78:69-108.

26. Wood MD, Wren PB. Serotonin-dopamine interactions: implications for the design of novel therapeutic agents for psychiatric disorders. Prog Brain Res. 2008;172:213-230.

27. Castagne V, Moser PC, Porsolt RD. Preclinical behavioral models for predicting antipsychotic activity. Adv Pharmacol. 2009;57:381-418.

28. Muir WJ, St Clair DM, Blackwood DH, Roxburgh $\mathrm{HM}$, Marshall I. Eye-tracking dysfunction in the affective psychoses and schizophrenia. Psychol Med. 1992;22(3):573-580.

29. de Visser SJ, van der Post J, Pieters MS, Cohen AF, van Gerven JM. Biomarkers for the effects of antipsychotic drugs in healthy volunteers. Br J Clin
Pharmacol. 2001;51(2):119-132.

30. Järup L. Hazards of heavy metal contamination. $\mathrm{Br}$ Med Bull. 2003;68:167-182.

31. Baloh RW, Langhofer L, Brown CP, Spivey GH. Quantitative eye tracking tests in lead workers. Am J Ind Med. 1980;1(1):109-113.

32. Specchio LM, et al. Smooth pursuit eye movements among storage battery workers. Clin Toxicol. 1981; 18(11):1269-1276.

33. Mameli $\mathrm{O}$, et al. Neurotoxic effect of lead at low concentrations. Brain Res Bull. 2001;55(2):269-275.

34. Klaasen CD. Heavy metals and heavy metal antagonists. In: Hardman JG, Limbird LE, eds. Goodman and Gilman's The Pharmacoloogic Basis of Therapeutic. 10th ed. New York, New York, USA: McGraw Hill; 2001:1851-1875

35. Blekher T, et al. Saccades in presymptomatic and early stages of Huntington disease. Neurology. 2006; 67(3):394-399.

36. Peltsch A, Hoffman A, Armstrong I, Pari G, Munoz DP. Saccadic impairments in Huntington's disease. Exp Brain Res. 2008;186(3):457-469.

37. Blekher $\mathrm{T}$, et al. Test-retest reliability of saccadic measures in subjects at risk for Huntington disease. Invest Ophthalmol Vis Sci. 2009;50(12):5707-5711.

38. Mangiarini L, et al. Exon 1 of the HD gene with an expanded CAG repeat is sufficient to cause a progressive neurological phenotype in transgenic mice. Cell. 1996;87(3):493-506.

39. Kuhn A, et al. Mutant huntingtin's effects on striatal gene expression in mice recapitulate changes observed in human Huntington's disease brain and do not differ with mutant huntingtin length or wild-type huntingtin dosage. Hum Mol Genet. 2007; 16(15):1845-1861.

40. Mancall EM. Essentials of the Neurologic Examination. 2nd ed. Philadelphia, Pennsylvania, USA: F.A. Davis; 1982.

41. Simpson JI. The accessory optic system. Annu Rev Neurosci. 1984;7:13-41.

42. Kitazawa H, Katoh A, Yagi T, Nagao S. Dynamic characteristics and adaptability of reflex eye movements of Fyn-kinase-deficient mice. Neurosci Lett. 2000;280(3):179-182

43. Yoshida K, Watanabe D, Ishikane H, Tachibana M,
Pastan I, Nakanishi S. A key role of starburst amacrine cells in originating retinal directional selectivity and optokinetic eye movement. Neuron. 2001; 30(3):771-780.

44. Iwakabe H, Katsuura G, Ishibashi C, Nakanishi S. Impairment of pupillary responses and optokinetic nystagmus in the mGluR6-deficient mouse. Neuropharmacology. 1997;36(2):135-143.

45. Ye X, et al. Norrin, frizzled-4, and Lrp5 signaling in endothelial cells controls a genetic program for retinal vascularization. Cell. 2009;139(2):285-298.

46. Badea TC, Cahill H, Ecker J, Hattar S, Nathans J. Distinct roles of transcription factors brn3a and brn3b in controlling the development, morphology, and function of retinal ganglion cells. Neuron. 2009; 61(6):852-864.

47. Diefendorf AR, Dodge R. An experimental study of the ocular reactions of the insane from photographic records. Brain. 1908;31(3):451-489.

48. Baldessarini RJ, Tarazi FI. Drugs and the treatment of psychiatric disorders. In: Hardman JG, Limbird LE, eds. Goodman and Gilman's The Pharmacoloogic Basis of Therapentic. 10th ed. New York, New York, USA: McGraw Hill; 2001:485-520.

49. Jones AK, Buckingham SD, Sattelle DB. Chemistryto-gene screens in Caenorhabditis elegans. Nat Rev Drug Discov. 2005;4(4):321-330.

50. Segalat L. Invertebrate animal models of diseases as screening tools in drug discovery. ACS Chem Biol. 2007;2(4):231-236

51. Winter MJ, Redfern WS, Hayfield AJ, Owen SF, Valentin JP, Hutchinson TH. Validation of a larval zebrafish locomotor assay for assessing the seizure liability of early-stage development drugs. J Pharmacol Toxicol Methods. 2008;57(3):176-187.

52. Rihel J, et al. Zebrafish behavioral profiling links drugs to biological targets and rest/wake regulation. Science. 2010;327(5963):348-351.

53. Roth BL, Sheffler DJ, Kroeze WK. Magic shotguns versus magic bullets: selectively non-selective drugs for mood disorders and schizophrenia. Nat Rev Drug Discov. 2004;3(4):353-359.

54. Heidbreder CA, Hagan JJ. Novel pharmacotherapeutic approaches for the treatment of drug addiction and craving. Curr Opin Pharmacol. 2005;5(1):107-118. 